\title{
Combinatory therapy targeting mitochondrial oxidative phosphorylation improves efficacy of IDH mutant inhibitors in acute myeloid leukemia
}

Lucille Stuani ${ }^{1}$, Marie Sabatier ${ }^{1}$, Feng $\mathrm{Wang}^{2}$, Nathalie Poupin ${ }^{3}$, Claudie Bosc ${ }^{1}$, Estelle Saland $^{1}$, Florence Castelli ${ }^{4}$, Lara Gales ${ }^{5,6}$, Camille Montersino ${ }^{7}$, Emeline Boet ${ }^{1}$, Evgenia Turtoi $^{8,9,10}$, Tony Kaoma ${ }^{11}$, Thomas Farge ${ }^{1}$, Nicolas Broin ${ }^{1}$, Clément Larrue ${ }^{1}$, Natalia Baran ${ }^{2}$, Marc Conti $^{12}$, Sylvain Loric ${ }^{12}$, Pierre-Luc Mouchel ${ }^{1,13}$, Mathilde Gotanègre ${ }^{1}$, Cédric Cassan ${ }^{14}$, Laurent Fernando $^{3}$, Guillaume Cognet ${ }^{1}$, Aliki Zavoriti ${ }^{1}$, Mohsen Hosseini ${ }^{1}$, Héléna Boutzen ${ }^{1}$, Kiyomi Morita ${ }^{2}$, Andrew Futreal ${ }^{2}$, Emeline Chu-Van $^{4}$, Laurent Le Cam ${ }^{9,10}$, Martin Carroll ${ }^{15}$, Mary A. Selak ${ }^{15}$, Norbert Vey ${ }^{16}$, Claire Calmettes ${ }^{17}$, Arnaud Pigneux ${ }^{17}$, Audrey Bidet ${ }^{18}$, Rémy Castellano $^{7}$, Francois Fenaille ${ }^{4}$, Andrei Turtoi ${ }^{8,9,10}$, Guillaume Cazals ${ }^{19}$, Pierre Bories ${ }^{20}$, Yves Gibon $^{14}$, Brandon Nicolay ${ }^{21}$, Sébastien Ronseaux ${ }^{21}$, Joe Marszalek ${ }^{2}$, Courtney D. DiNardo ${ }^{2}$, Marina Konopleva ${ }^{2}$, Yves Collette ${ }^{7}$, Laetitia K. Linares ${ }^{9,10}$, Floriant Bellvert ${ }^{5,6}$, Fabien Jourdan $^{3,6}$, Koichi Takahashi ${ }^{2}$, Christian Récher ${ }^{1,13}$, Jean-Charles Portais ${ }^{5,6}$, Jean-Emmanuel Sarry $^{1 *}$

${ }^{1}$ Centre de Recherches en Cancérologie de Toulouse, UMR1037, Inserm, Université de Toulouse 3 Paul Sabatier, Equipe Labellisée LIGUE 2018, F-31037 Toulouse, France.

${ }^{2}$ Departments of Leukemia and Genomic Medicine, The University of Texas, MD Anderson Cancer Center, Houston, TX 77030, USA.

${ }^{3}$ UMR1331 Toxalim, Université de Toulouse, INRA, ENVT, INP-Purpan, UPS, Toulouse, France.

${ }^{4} \mathrm{CEA} / \mathrm{DSV} / \mathrm{BBiTec-S} / \mathrm{SPI}$, Laboratoire d'Etude du Métabolisme des Médicaments, MetaboHUB-Paris, F-91191 Gif-sur-Yvette, France.

${ }^{5}$ TBI, Université de Toulouse, CNRS, INRA, INSA, Toulouse, F-31077, France.

${ }^{6}$ MetaToul-MetaboHUB, National Infrastructure of Metabolomics and Fluxomics, Toulouse, F-31077, France.

${ }^{7}$ Aix-Marseille Univ, Inserm, CNRS, Institut Paoli-Calmettes, CRCM, Marseille, France.

${ }^{8}$ Inserm, U1194, Cancer Research Institute of Montpellier, Tumor Microenvironment Lab, F34090 Montpellier, France

${ }^{9}$ Institut du Cancer de Montpellier, F-34090 Montpellier, France

${ }^{10}$ Université de Montpellier, F-34090 Montpellier, France.

${ }^{11}$ Proteome and Genome Research Unit, Department of Oncology, Luxembourg Institute of Health, L-1445 Strassen, Luxembourg.

${ }^{12}$ Inserm U955EQ7, IMRB, Créteil, France.

${ }^{13}$ Service d'Hématologie, Institut Universitaire du Cancer de Toulouse-Oncopole, CHU de Toulouse, F-31100 Toulouse, France.

${ }^{14}$ UMR1332 Biologie du Fruit et Pathologie, Plateforme Métabolome Bordeaux, INRA, Université de Bordeaux, F-33883 Villenave d'Ornon, France.

${ }^{15}$ Division of Hematology \& Oncology, Department of Medicine, University of Pennsylvania, Philadelphia, PA 19104, USA.

${ }^{16}$ Institut Paoli-Calmettes and Aix-Marseille Université, Marseille, France.

${ }^{17} \mathrm{CHU}$ Bordeaux, Service d'Hématologie Clinique, F-33000 Bordeaux, France.

${ }^{18} \mathrm{CHU}$ Bordeaux, Service d'Hématologie Biologique, F-33000 Bordeaux, France.

${ }^{19}$ Laboratoire de Mesures Physiques, Université de Montpellier, F-34090 Montpellier, France.

${ }^{20}$ Réseau régional de Cancérologie Onco-Occitanie, Toulouse, France.

${ }^{21}$ Agios Pharmaceuticals, 88 Sydney St, Cambridge MA, USA. 
49 *Corresponding author: Jean-Emmanuel Sarry; Inserm, U1037, Centre de Recherches en

50 Cancérologie de Toulouse, F-31024 Toulouse cedex 3, France; Email: jean-

51 emmanuel.sarry@inserm.fr; Phone:+33 582741632

52

53 Running Title: IDH and OxPHOS inhibitors

54 
56 Isocitrate dehydrogenases (IDH) are involved in redox control and central metabolism.

57 Mutations in IDH induce epigenetic and transcriptional reprogramming, differentiation

58 bias, BCL-2 dependence and susceptibility to mitochondrial inhibitors in cancer cells.

59 Here we show that high sensitivity to mitochondrial oxidative phosphorylation

60 (OxPHOS) inhibitors is due to an enhanced mitochondrial oxidative metabolism in cell

61 lines, PDX and patients with acute myeloid leukemia (AML) harboring IDH mutation.

62 Along with an increase in TCA cycle intermediates, this AML-specific metabolic

63 behavior mechanistically occurs through the increase in methylation-driven CEBP $\alpha$ -

64 and CPT1a-induced fatty acid oxidation, electron transport chain complex I activity and

65 mitochondrial respiration in IDH1 mutant AML. Furthermore, an IDH mutant

66 inhibitor that significantly and systematically reduces 2-HG oncometabolite transiently

67 reverses mitochondrial FAO and OxPHOS gene signature and activities in patients who

68 responded to the treatment and achieved the remission. However, at relapse or in

69 patients who did not respond, IDH mutant inhibitor failed to block these mitochondrial

70 properties. Accordingly, OxPHOS inhibitors such as IACS-010759 improve anti-AML

71 efficacy of IDH mutant inhibitors alone and in combination with chemotherapy in vivo.

72 This work provides a scientific rationale for combinatory mitochondrial-targeted

73 therapies to treat IDH mutant-positive AML patients, especially those unresponsive to

74 or relapsing from IDH mutant-specific inhibitors. 
77 Changes in intermediary and energy metabolism provide the flexibility for cancer cells to 78 adapt their metabolism to meet energetic and biosynthetic requirements for proliferation ${ }^{1-4}$.

79 Manipulating glycolysis, glutaminolysis, fatty acid $\beta$-oxidation (FAO) or oxidative 80 phosphorylation (OxPHOS) markedly reduces cell growth in vitro and in vivo and sensitizes 81 acute myeloid leukemia (AML) cells to chemotherapeutic drugs ${ }^{5-13}$. The importance of the 82 metabolic reprogramming in this disease is further illustrated by recurrent mutations in genes 83 of two crucial metabolic enzymes, isocitrate dehydrogenases (IDH) 1 and 2, present in more 84 than $15 \%$ of AML patients ${ }^{14-17}$.

85 The impact of IDH mutation and the related accumulation of the oncometabolite (R)-286 hydroxyglutarate (2-HG) have been well documented in leukemic transformation and AML 87 biology ${ }^{18-28}$. As IDH mutations are early events in oncogenesis and are systematically 88 conserved at relapse ${ }^{29-31}$, IDH1/2 mutated (IDHm) enzymes represent attractive therapeutic 89 targets and small molecules specifically inhibiting the mutated forms of these enzymes have 90 been developed and recently approved by the FDA $^{32-41}$. Both the IDH2m- and IDH1m91 inhibitors promote differentiation and reduce methylation levels as well as significantly 92 decrease 2-HG levels $33,36,37,42,43$. Overall response rates for ivosidenib (IDH1m inhibitor) and 93 enasidenib (IDH2m inhibitor) are highly encouraging up to 30 or $40 \%$ in monotherapy in 94 phase I/II clinical trials for newly diagnosed or relapsed/refractory AML patients 95 respectively). However, several mechanisms of resistance to these targeted therapies have 96 been already identified ${ }^{37-39,42,44}$. Moreover, suppression of serum 2-HG level alone did not 97 predict response in patients, as many non-responders also displayed a significant decrease in 98 the amount of $2-\mathrm{HG}^{37,40,42,44-47}$. Importantly, multiple pathways involved in signaling, clonal 99 heterogeneity or second-site mutation are very recently considered to be responsible for 100 relapses in patients treated with IDH mutant inhibitors ${ }^{38,48-50}$. Thus, targeting IDH mutant 
101 activity is not sufficient to achieve a durable clinical response in most patients and new 102 combinatory approaches need to be designed.

103 Given the crucial roles of wild type (WT) IDH1/2 in cell metabolism (e.g. Krebs cycle, 104 OxPHOS, cytosolic and mitochondrial redox, anabolism including lipid biosynthesis) and in 105 human disease ${ }^{51}$, a better understanding of the contribution of oncogenic IDH mutations to 106 metabolism and metabolic homeostasis is expected to lead to new therapeutic strategies. 107 Several studies have demonstrated that IDH mutant cancer cells exhibit some metabolic 108 specificities ${ }^{52-58}$. However, none of these studies have definitively shown how metabolic 109 changes elicited by IDH mutations modulate cell proliferation and drug resistance or impact 110 therapeutic response in AML. In particular, the role of metabolism in resistance to IDHm 111 inhibitors has not been yet comprehensively studied in AML. Although existing literature in 112 the field described several vulnerabilities to mitochondrial inhibitors in IDH1/2-mutant cells 113 from solid tumors and $\mathrm{AML}^{9,59-62}$, no studies have also fully demonstrated why IDH mutant 114 cells are more sensitive to mitochondrial inhibitors in AML. We therefore hypothesized that 115 mitochondrial oxidative phosphorylation plays a crucial role in IDH mutant biology and in the 116 response of AML patients with IDH mutation to IDHm inhibitors.

117 In the present study, we perform multi-omics and functional approaches using 2 118 engineered AML cell lines, 12 PDX models from two clinical sites (Toulouse Hospital and 119 University of Pennsylvania) and 123 patient samples from four clinical sites (Toulouse 120 Hospital TUH, Bordeaux Hospital BUH, Marseille Hospital IPC and MD Anderson Cancer 121 Center MDACC) to test this hypothesis and to expressly understand the mitochondrial 122 reprogramming induced by IDH1 mutation and its role in the response to IDH mutant specific 123 inhibitors.

\section{Results}


126 A higher susceptibility of IDH1 mutant AML cells to mitochondrial inhibitors is due to

127 their enhanced OxPHOS activity. First we confirmed a higher sensitivity of IDH1/2-mutant

128 cells from primary AML patient samples (WT, $n=64 ;$ MUT, $n=56 ;$ TUH, BUH, IPC,

129 MDACC; Supplementary Table 1) and two genetically diverse cell lines to mitochondrial

130 inhibitors such as OxPHOS inhibitors, including a new electron transport chain (ETC)

131 complex I inhibitor IACS- $010759^{63}$ and metformin, ETC complex III (antimycin A, AA;

132 atovaquone, ATQ), ETC complex V (oligomycin, OLIGO) or BCL2 inhibitors (ABT-199,

133 ABT-263) (Fig. 1a-c and Supplementary Fig. 1a). Interestingly, their 2-HG response was

134 heterogeneous (Fig. 1d). Whereas metformin induced an increase in 2-HG content, the BCL2

135 inhibitor ABT-199 caused a reduction in the amount of the oncometabolite. This strongly

136 suggests an enhancement of mitochondrial metabolic dependency in IDH mutant subgroup of

137 AML patients without a systematic correlation with 2-HG content.

138 To better understand why IDH1 mutant cells have a higher sensitivity to mitochondrial

139 inhibition, we extensively analyzed several biochemical, enzymatic and functional features

140 relative to mitochondrial activity in IDH1 mutant versus WT AML cells from two genetically

141 diverse AML cell lines in vitro and six patient-derived xenografts in vivo (Supplementary Fig.

142 1b). Mitochondrial membrane potential, oxygen consumption, ATP-linked respiration and

143 ATP content were all significantly enhanced in IDH mutant AML cells in vitro and in vivo

144 (Fig. 1e-g and Supplementary Fig. 1c-e). Importantly, ETCI complex (and not other ETC 145 complexes) activity, NADH-producing enzyme activity of TCA enzymes such as malate 146 dehydrogenase (MDH2) and isocitrate dehydrogenase (IDH3) and concentration of Krebs 147 cycle intermediates (except $\alpha-K G$ ) were also increased in IDH1 mutant AML cells (Fig. 1h-j), 148 indicating an increase in mitochondrial $\mathrm{NADH}$ availability, mitochondrial activities and 149 OxPHOS dependency specifically in IDH1 mutant AML cells. Interestingly, this was not due 150 to an increase in mitochondrial biogenesis as shown by mitochondrial mass, protein content of 
151 ETC complexes, citrate synthase activity or ratio between mitochondrial and nucleic DNA, 152 which were not affected (Supplementary Fig. 1f-j).

153 Drug-resistant cancer cells have recently shown to be enriched in cells exhibiting a high

154 OxPHOS signature and enhanced mitochondrial function in several cancers including myeloid

155 malignancies ${ }^{11,12,64,65}$. Accordingly, we observed that IDH1 mutant cells were more resistant

156 to conventional cytarabine (AraC) chemotherapy than IDH1 WT cells in vitro and in three 157 PDX models in vivo that are low responders (Fig. 1k and Supplementary Fig. 1k), as 158 previously defined to distinguish patients with high from low $\mathrm{AraC}$ response in vivo ${ }^{11}$.

159

Methylation- and CEBP $\alpha$ - dependent mitochondrial FAO is increased in IDH1 mutant

161 cells. In order to further identify the mitochondrial reprogramming induced by IDH1 162 mutation, we next performed a computational analysis of the metabolic network of IDH1 163 mutant cells based on human genome scale metabolic network reconstruction Recon2 (7440 164 metabolic reactions) $)^{66}$. To reconstruct active leukemic metabolic networks of IDH1 WT and 165 mutant AML cells at a global level, we integrated transcriptomic data and applied metabolic 166 constraints according to metabolite production and consumption rates measured in the 167 corresponding cell culture supernatants (exometabolome) ${ }^{67,68}$ (Fig. 2a). This analysis 168 identified a significant enrichment of active reactions in various carbon metabolic pathways 169 (N-glycan synthesis, fructose and mannose metabolism, dicarboxylate metabolism; Fig. 2a) in 170 IDH1 mutant cells and predicted a major change in FAO in these cells (Fig. 2a-b), especially 171 CPT1 which is required to initiate the transfer of fatty acids from the cytosol to the 172 mitochondrial matrix for oxidation (Supplementary Fig. 2a). This prediction prompted us to 173 assess key features of FA utilization in AML cells and patients with IDH1 mutation. First, we 174 measured acyl-CoAs as readout of FA catabolism. As expected, acetyl-CoA, succinyl-CoA, 
175 free coenzyme A and FA oxidation rate were significantly increased in IDH1 mutant AML 176 cells (Fig. 2c-d).

177 The regulation of FAO is complex and involves different signaling pathways and allosteric 178 regulation (Supplementary Fig. 2a). We first examined AMP kinase (AMPK), a master 179 regulator of energy and metabolic homeostasis. Surprisingly, whereas the AMPK protein 180 level was increased in IDH1 mutant cell lines and patients, its activation by phosphorylation 181 was not increased (Fig. 2b). Furthermore, we did not observe an increase in the AMP/ATP 182 ratio in mutants compared to WT (Supplementary Fig. 2c), suggesting that AMP, the primary 183 allosteric activator of AMPK, does not directly activate AMPK in these cells. No increase in $184 \mathrm{ADP} / \mathrm{ATP}$ ratio was detected, suggesting that neither AMP nor ADP enhanced the canonical 185 phosphorylation of AMPK Thr172 via LKB1 in IDH1 mutant AML cells (Supplementary Fig. 186 2c). These data showed that AMPK is not activated directly by AMP or via phosphorylation 187 in mutant cells, indicating that the changes seen in FAO in mutant cells reflect an AMPK188 independent mechanism. Similarly, the PKA pathway did not appear to be differentially 189 activated and to be involved into the FAO regulation (Supplementary Fig. 2d). Interestingly, 190 protein levels and phosphorylation of both acetyl-CoA carboxylases (ACC1 and ACC2) that 191 regulate malonyl-CoA level and hence modulate mitochondrial FA shuttling and oxidation, 192 were decreased in IDH1 mutant AML cells and therefore favor FAO (Supplementary Fig. 2e). 193 Transcriptomic data using two independent AML cohorts (GSE $14468^{69}$ and TCGA ${ }^{70}$ ) 194 reinforced this observation demonstrating a significant decrease in $A C A C A$ (coding ACC1) 195 and $A C A C B$ (coding ACC2) mRNA expression in IDH1 mutant cells (Supplementary Fig. 2f).

196 Moreover, previous reports have shown that gene expression is closely modulated by histone 197 and DNA methylation in IDH mutant cells ${ }^{18,71,72}$. To address the importance of histone 198 methylation at the $A C A C A$ promoter, we performed quantitative chromatin 199 immunoprecipitation (qChIP) experiments to measure the levels of trimethylation of lysine 4 
200 of histone $\mathrm{H} 4$ (H3K4me3) and of trimethylation of lysine 27 of histone H3 (H3K27me3), two

201 epigenetic markers associated with transcriptional activation and repression, respectively.

202 While we did not observe any differences in H3K4me3 occupancy of the ACACA promoter, a

203 significant increase in $\mathrm{H} 3 \mathrm{~K} 27 \mathrm{me} 3$ on $A C A C A$ occurred specifically in IDH1 mutant cells

204 (Supplementary Fig. 2g). Furthermore, we performed a gene set enrichment analysis (GSEA)

205 with a curated FAO gene signature ${ }^{73}$ and found that this signature was enriched in IDH1

206 mutant AML cells (Supplementary Fig. 2h). The most highly expressed gene in IDH1 mutant

207 cells was the key component of FA shuttling into mitochondria CPT1a. Consistent with this,

$208 C P T 1 a$ and its isoform $C P T 1 b$ mRNA levels were also significantly upregulated in our IDH1

209 mutant cell lines and in two independent AML cohorts (Fig. 2e and Supplementary Fig. 2i).

210 Also, CPT1A protein was significantly increased in total cell lysates and in mitochondria

211 isolated from IDH1 mutant cells compared to IDH1 WT cells and in IDH1 mutant primary

212 samples (Fig. 2f). Finally, GSEA analysis comparing the transcriptomes of AML patients

213 with IDH WT, IDH1 or IDH1/2 mutation revealed higher enrichment of FA metabolism and

214 OxPHOS gene signatures in $\mathrm{CPT} 1 \mathrm{a}^{\mathrm{HIGH}}$ patients with IDH mutations in two-independent

215 cohorts (Fig. 2g). This strengthens the observation that CPT1a plays a crucial role in FA

216 metabolism and OxPHOS in IDH mutant AML cells.

217 We previously demonstrated that IDH1 mutation and its 2-HG product dysregulate

$218 \mathrm{CEBP} \alpha^{23}$, a well-known transcriptional regulator of several genes involved in glucose and

219 lipid metabolism. Moreover, CEBP $\alpha$ was the second most highly expressed gene of the FAO

220 gene signature in IDH1 mutant cells (Supplementary Fig. 2h). Thus, we performed qChIP

221 assays to assess CEBP $\alpha$ binding to promoters of genes encoding FA transporters. We

222 observed that the recruitment of endogenous CEBP $\alpha$ to promoter of CPTIa, CPT2, and

223 SLC25A20 that mediates the transport of acyl-carnitines of different length across the

224 mitochondrial inner membrane from the cytosol to the mitochondrial matrix, increased 
225 specifically in IDH1 mutant cells (Fig. 2h). Furthermore, CEBP $\alpha$ silencing led to a reduction

226 of mitochondrial basal OCR as well as ATP-linked and FAO-coupled OCR in IDH1 WT and

227 to a greater extent in IDH1 mutant AML cells (Supplementary Fig. 2j-k). Together, these

228 results indicate that IDH1 mutant cells display a gene signature specific for FA shuttling and a

229 high FAO activity in CPT1a- and $\mathrm{CEBP} \alpha$ - dependent manner to support mitochondrial 230 activity.

Reduction of 2-HG with IDHm inhibitors transiently reverses the mitochondrial phenotype of IDH mutant AML cells. As IDH mutant cells exhibited higher mitochondrial activity than WT cells, we investigated the impact of IDHm inhibitors (FDA approved ivosidenib AG-120 and its preclinical version AGI-5198 for IDH1 and enasidenib AG-221 for IDH2) on this OxPHOS phenotype. Because Phase I clinical trials NCT02074839 or

237 NCT01915498 showed 40\% overall response rate for ivosidenib or enasidenib monotherapy 238 for IDH mutant AML patients with relapsed or refractory AML, respectively ${ }^{38,39}$, we reasoned 239 that non-responders in this study might have different mitochondrial and FAO status. 240 Interestingly, we performed comparative transcriptomic analyses of IDH mutant patients 241 characterized as good responders to IDHm inhibitor at complete remission (bone marrow 242 blast $<5 \%$ and normalization to peripheral blood count; $\mathrm{n}=6$ patients) versus before treatment 243 and at relapse post-IDHm inhibitor versus at complete remission. We thus shown that curated 244 gene signature related to OxPHOS, Krebs cycle, FAO and pyruvate metabolism were 245 enriched in patients before treatment and upon relapse (Fig. 3a). Furthermore, these gene 246 signatures were significantly enriched after IDHm inhibitor treatment in two IDH mutant 247 patients who did not respond to IDHm inhibitor (Fig. 3a). In particular, the expression of the 248 FAO genes CPT1a, CPT2, SLC25A20 was significantly reduced in good responders to IDHm 249 inhibitor at complete remission compared to before treatment but then increased at relapse to 
250 reach the same level as before treatment (Fig. 3b). In two AML cell lines harboring IDH1

251 mutation, AG-120 and AGI-5198 treatments significantly reduced 2-HG levels and decreased

252 the expressions of CEBPa, CPT1a, CPT2 and SLC25A20 (Supplementary Fig. 3a-b).

253 Furthermore, IDH1m inhibitors prevented the recruitment of endogenous CEBP $\alpha$ to promoter

254 of CPT1a, CPT2 and SLC25A20 (Supplementary Fig. 3c). As FAO is one of the major

255 biochemical pathways that support OxPHOS and mitochondrial function especially in

$256 \mathrm{AML}^{5,11,74}$, it was surprising to observe that IDH1m inhibitor maintained or even increased

257 FAO-coupled (Fig. 3c), basal mitochondrial (Fig. 3d) and ATP-linked OCR (Fig. 3e). We

258 then assessed several mitochondrial activities after treatment with IDHm inhibitors. IDH1m

259 inhibitors also maintained or increased mitochondrial response in both IDH1 mutant cell lines

260 such as their mitochondrial membrane potential (Supplementary Fig. 3d), TCA cycle

261 intermediate concentrations (Fig. 3f), ETC complex activities or protein amounts

262 (Supplementary Fig. 3e-g). Altogether, these results demonstrated that, while decreasing the

263 level of 2-HG and some FAO features, IDHm inhibitors only transiently reverse OxPHOS

264 phenotype, leading us to specifically consider combination with OxPHOS inhibitor for these 265 patients.

267 Treatment with OxPHOS inhibitors enhances anti-leukemic effects of IDH-mutant 268 specific inhibitors alone and in combination with cytarabine. We assessed several mono, 269 duplet or triplet therapeutic approaches in vivo with IDHm inhibitor AG-120 (150 mg/kg, 270 twice every day, 3 weeks), ETC complex I inhibitor IACS-010759 (8 mg/kg, every other day, 2713 weeks) or/and AraC (30 mg/kg, every day, 1 week) in two IDH1 R132 PDX models with 272 variable engraftment capacity (high engrafting patient \#325 in Fig. 4a-b, Supplementary Fig. 273 4; low engrafting patient \#1065 in Supplementary Fig. 5a). The level of 2-HG was greatly 274 reduced in the PDX sera upon all therapies compared to control group while $\alpha-\mathrm{KG}$ remained 
275 unchanged in PDX \#325 (Fig 4c and Supplementary Fig. 4a). Total cell tumor burden was

276 significantly reduced in mono, duplet and triplet therapy with a greater effect in the triplet 277 therapy cohort compared to vehicle cohort or AraC monotherapy (Fig. 4d). Similarly, 278 apoptosis was also increased in all treatments except AG-120 mono and duplet therapy with 279 IACS and we observed a greater significance in the triplet therapy compared to vehicle 280 (Supplementary Fig. 4b). Expression of the myeloid differentiation marker CD15 was also 281 significantly increased in duplet therapy combining AraC and IACS and even more in the 282 triplet therapy (Fig 4e and Supplementary Fig. 4c). Interestingly, mitochondrial OxPHOS 283 function assessed in vivo by mitochondrial membrane potential was only decreased in the 284 triplet therapy (Supplementary Fig. 4d). Furthermore, mitochondrial ATP content and 285 respiratory capacities were increased after AraC or AG-120 alone or combined together but 286 rescued with the addition of IACS in the triplet therapy in vivo (Fig. 4f and Supplementary 287 Fig. 4e-g). Analysis of mice serum metabolomes showed that aspartate level was significantly 288 reduced in all AraC groups, in particular in the duplet therapy with AG-120 and the triplet 289 therapy. Lactate level was enhanced in all groups with IACS including the triplet therapy as 290 key biomarker of IACS-010759 response (Fig. 4g). Similar in vivo experiments with lower 291 engrafting IDH1-R132 PDX showed a lower level in anti-leukemic and biological effects of 292 the mono, duplet and triplet combinations (Supplementary Fig. 5). However and more 293 importantly in this low responder PDX, the triplet therapy induces a greater decrease in the 294 total cell tumor burden, in mitochondrial activity through decreased mitochondrial membrane 295 potential, mitochondrial ATP and enhanced lactate amount in mice sera (Supplementary Fig. $2965 b-e)$. Of note, global toxicity of the triplet therapy or duplet therapies with AraC was 297 primarily driven by AraC toxicity (Supplementary Fig. 4h-i and Fig. 5f-g). Altogether, these 298 results not only confirmed that IDHm inhibitor does not necessarily reverse metabolic and 299 mitochondrial (especially, enhanced OxPHOS phenotype) features of IDH mutant cells in 
300 vivo but also that combining this drug with ETC complex I inhibitor in presence or not of 301 standard AraC chemotherapy increases its drug efficacy in vivo, notably by inducing a Pasteur 302 effect (e.g. increased lactate in response to the inhibition of mitochondrial ATP production). 303 Finally, taking advantage of this preclinical study, we have identified a set of metabolic 304 changes (here called AML metabolic profiling) that represent a combination of classic 305 hallmarks of the Pasteur effect and other metabolic adaptations to predict the response to IDH 306 plus OxPHOS inhibitors (Supplementary Fig. 6 and Supplementary Table 2) and to monitor 307 the efficacy of their response (Supplementary Table 3) in IDH mutant AML subgroup.

Discussion

310 Since the discovery of IDH mutations in various cancers, significant efforts have been 311 directed to understand the extent to which these oncogenic mutations directly impact 312 metabolism, histone/DNA methylation and gene expression ${ }^{18,26,27,75-78}$, cell proliferation and 313 differentiation bias $^{19,21-23,34}$. However, questions remain unanswered related to the impact of 314 IDH mutation on mitochondrial energetic metabolism in IDH mutant cells. Here we address 315 several aspects of these different crucial points in IDH mutant cell biology and explain 316 mitochondrial dependency in the basal condition and upon IDHm inhibitor treatment. IDH 317 mutation induces mitochondrial reprogramming that contributes to maintain the pool of $\alpha-\mathrm{KG}$ 318 to support 2-HG production and to replenish other Krebs cycle intermediates necessary for 319 anabolic reactions, oxygen consumption and ATP production by oxidative phosphorylation in 320 AML (Fig. 4h). Importantly, treatment with IDHm inhibitors transiently reverses FAO and 321 OxPHOS activities that are maintained or enhanced in non-responders or at relapse (Fig. 4h).

322 Several studies in different cancers have consistently shown increased mitochondrial 323 phenotypes in IDH mutant cells ${ }^{59,61,79,80}$. Our study helps to explain the dependence of IDH 324 mutant cells on mitochondria and energetic metabolism required to sustain mutant cell 
325 proliferation, in particular for synthesis of $\alpha-K G$ and NADPH, the substrates of mutant IDH 326 enzyme activity. It is noteworthy that disturbances in cellular and mitochondrial metabolism 327 can contribute to the chemoresistance of AML cells ${ }^{5,6,11}$. Here we showed that IDH1 mutant 328 AML cells exhibited a higher OxPHOS phenotype than WT cells, consistent with a lower 329 response to AraC. We also observed a OxPHOS hyperactivity of IDH mutant cells and 330 confirmed a strong ETC1 complex dependency in AML ${ }^{7,11,63,81,82}$. Accordingly, mutant AML 331 cells exhibit enhanced vulnerabilities to various small molecules targeting mitochondrial 332 OxPHOS such as inhibitors of ETC complex I, III and V. Consistent with literature reports 333 and current clinical trials, we also observed that IDHm AML cells were more sensitive to 334 BCL2 inhibition by ABT-199 20,62,83. Several combinations of BCL2i with newly approved 335 targeted therapies such as FLT3i and IDHi are under clinical assessment (NCT03735875 and 336 NCT03471260, respectively). Interestingly, 2-HG levels did not correlate with apoptosis in 337 these experiments, as its concentration decreased after ABT-199 while it increased after 338 metformin treatment, a result already observed in IDH1 R132H transformed mammary 339 epithelial cells ${ }^{61}$. This lack of correlation between cellular concentration of 2-HG, cell 340 survival and sensitivity to various inhibitors is of particular interest as it has been shown that 341 neither did inhibitors of mutant IDH reverse all IDH-mutant phenotypes nor did suppression 342 of 2-HG alone predict response to IDHm inhibitors ${ }^{33,44,45,59,84,85}$. Our data and previous reports 343 clearly show that $2-\mathrm{HG}$ is essential but insufficient in mediating and mimicking all the 344 complex metabolic consequences of IDH mutation ${ }^{56,86}$. These observations strongly suggest 345 that innovative combinatory therapies might be useful in this patient subgroup. Of particular 346 interest, whereas IDHm inhibitors maintain or increase mitochondrial and OxPHOS activity 347 in IDH mutant cell lines and primary patients in vitro, in vivo and in relapsed or refractory 348 AML patients in clinical trials, triplet combination using IDH1m inhibitor, OxPHOS inhibitor 349 (such as IACS-010759), and AraC showed promising effects in IDH1 mutant AML PDX. 
350 Therefore, this triplet drug combination may represent a beneficial alternative for AML

351 patients unresponsive to IDH mutant-specific inhibitors.

352 In this regard, transcriptomic analysis before administration of an IDHm inhibitor in AML

353 patients with IDH mutation, at complete remission and at relapse and in patients who did not

354 respond to the drug revealed an enrichment in FAO and OxPHOS gene signatures at relapse 355 and in non-responders. In particular, expression of several genes participating in FAO such as 356 CPT1a, CPT2 and SLC25A20 correlate with relapse and response to IDHm inhibitor and 357 could potentially be used in clinics to predict and monitor the therapeutic efficacy of IDHm 358 and OxPHOS inhibitors. Moreover, we observed that lactate concentration in mice sera was 359 not modified and that mitochondrial ATP was maintained or increased by treatment with 360 IDH1m inhibitor alone while duplet or triplet therapies with OxPHOSi lead to significant 361 increase in lactate concentration and reduction in mitochondrial ATP in our high responder 362 PDX harboring IDH1 mutation. Accordingly, we proposed an AML metabolic profiling based 363 on measuring mitochondrial ATP, OxPHOS and FAO gene signatures from primary cells, and 364 lactate, aspartate and 2-HG in the sera of patients. This could represent a potentially powerful 365 tool in identifying and understanding dependence on individual mitochondrial FAO and 366 OxPHOS activities. Consequently, this combination of metabolic and genetic approaches can 367 also be used to predict and monitor responses to the duplet or triplet therapy combining IDHi 368 and OxPHOSi (Supplementary Fig. 6, Supplementary Table 2 and Supplementary Table 3) in 369 the context of the functional precision cancer medicine ${ }^{87,88}$.

370 Of particular interest, it was very recently shown that for patients with IDH1 or IDH2 371 mutation who responded to IDHm inhibitors in clinics and then relapsed, acquired resistance 372 to this molecularly targeted therapy was caused by the emergence of clones with a second373 side IDH mutation in the wild type IDH allele without the initial IDH mutation, rescuing 2374 HG production ${ }^{48}$. This reinforced the therapeutic interest/potential of our combinatory 
375 strategy. Finally, our study supports the merit of future clinical trials testing the combination

376 of IDHm inhibitors and mitochondrial inhibitors with cytarabine treatment. Because this

377 proposed therapeutic strategy will overcome different newly identified mechanisms of

378 resistance to IDH mutant inhibitors ${ }^{38,48,49}$, this would be especially relevant as alternative

379 therapeutic approaches for the treatment of those patients that are not unresponsive to or

380 relapsing from IDH mutant-specific inhibitors.

381

382 Methods

383 Primary AML samples

384 Primary AML patient specimens are from five clinical sites [University of Pennsylvania

385 (UPENN), Philadelphia, PA; MD Anderson Cancer Center at University of Texas, Houston, 386 (MDACC), Toulouse University Hospital (TUH), Toulouse, France, Institut Paoli-Calmettes 387 (IPC), Marseille, France and Bordeaux University Hospital (BUH), Bordeaux, France].

388 For TUH and BUH, frozen samples of bone marrow or peripheral blood were obtained from 389 patients diagnosed with AML after signed informed consent in accordance with the 390 Declaration of Helsinki, and stored at the HIMIP collection (BB-0033-00060) and CRB-K 391 BBS (BB-0033-00036). According to the French law, HIMIP biobank collection and BBS 392 biobank have been declared to the Ministry of Higher Education and Research (DC 2008-307, 393 collection 1 for HIMIP and DC 2014-2164 for BBS) and obtained a transfer agreement (AC 394 2008-129) after approbation by the Comité de Protection des Personnes Sud-Ouest et 395 Outremer II (ethical committee). Clinical and biological annotations of the samples have been 396 declared to the CNIL (Comité National Informatique et Libertés ie Data processing and 397 Liberties National Committee). For IPC clinical site, ex vivo drug sensitivity was performed 398 on previously frozen (HEMATO-BIO-IPC 2013--015 clinical trial, NCT02320656) or fresh 399 (CEGAL-IPC-2014-012, NCT02619071 clinical trial) mononuclear cell samples from 49 
400 AML patients after informed consent (Supplementary Table 1). Both trials have been 401 approved by a properly constituted Institutional Review Board (Comité de Protection des 402 Personnes) and by the French National Security Agency of Medicine and Health Products 403 (ANSM). The samples are subjected to NGS to screen for mutations within a selected panel of $404 \sim 150$ actionable genes in AML (i.e.) known to be of prognostic value and/or druggable. For 405 UPENN, AML samples were obtained from patients diagnosed with AML in accordance with 406 U.S. Common Rules at the Stem Cell and Xenograft Core Facility at the UPENN School of 407 Medicine and with informed consent in accordance with institutional guidelines. Peripheral 408 blood or bone marrow samples were frozen in FCS with $10 \%$ DMSO and stored in liquid 409 nitrogen. The percentage of blasts was determined by flow cytometry and morphologic 410 characteristics before purification. For MDACC cohort, bone marrow samples were collected 411 from the 11 AML patients who were treated with AG-120 $(n=6)$ and AG-221 (n=5) in The 412 University of Texas MD Anderson Cancer Center from clinical trials NCT01915498 413 (enasidenib AG-221 for IDH2 mutated patients), NCT02074839 (ivosidenib AG-120 for 414 IDH1 mutated patients). All patients had provided written informed consent for sample 415 collection and subsequent data analysis. Nine patients had achieved complete remission 416 (responders; $\mathrm{n}=9$ ), and 2 patients never achieved remission (non-responders; $\mathrm{n}=2$ ). For a 417 subset of responders, longitudinal samples were obtained at pre-treatment $(n=8)$, complete 418 remission $(n=6)$, and at the time of relapse $(n=5)$. For non-responders $(n=2)$, we analyzed 419 the paired bone marrow samples obtained at pre-treatment and post-treatment. These samples 420 were analyzed by targeted capture next generation sequencing using SureSelect custom panel 421 of 295 genes (Agilent Technologies, Santa Clara, CA, USA), as well as RNA sequencing. 422 Bone marrow morphology and karyotyping data were interpreted by the board certified 423 hematopathologists.

\section{Mice and mouse xenograft model}


425 Animals were used in accordance with a protocol reviewed and approved by the Institutional

426 Animal Care and Use Committee of Région Midi-Pyrénées (France). NOD/LtSz-SCID/IL-

427 2Rychainnull (NSG) mice were produced at the Genotoul Anexplo platform at Toulouse

428 (France) using breeders obtained from Charles River Laboratories. Mice were housed in

429 sterile conditions using high-efficiency particulate arrestance filtered microisolators and fed

430 with irradiated food and sterile water.

431 Human primary AML cells were transplanted as reported previously ${ }^{11,89-91}$. Briefly, mice (6-

4329 weeks old) were sublethally treated with busulfan $(20 \mathrm{mg} / \mathrm{kg} / \mathrm{day}) 24$ hours before injection

433 of leukemic cells. Leukemia samples were thawed at room temperature, washed twice in PBS,

434 and suspended in Hank's Balanced Salt Solution at a final concentration of $0.2-10 \times 10^{6}$ cells

435 per $200 \mu \mathrm{L}$ of Hank's Balanced Salt Solution per mouse for tail vein injection. Transplanted

436 mice were treated with antibiotic (Baytril) for the duration of the experiment. Daily

437 monitoring of mice for symptoms of disease (ruffled coat, hunched back, weakness, and

438 reduced mobility) determined the time of killing for injected animals with signs of distress. If

439 no signs of distress were seen, mice were initially analyzed for engraftment 8 weeks after

440 injection except where otherwise noted.

\section{In vivo mice treatment}

442 Eight to 18 weeks (PDX) after AML cell transplantation and when mice were engrafted

443 (tested by flow cytometry on peripheral blood or bone marrow aspirates), NSG mice were

444 treated as described below:

445 - AraC treatment: NSG mice were treated by daily intraperitoneal injection of $60 \mathrm{mg} / \mathrm{kg}$

446 AraC for 5 days; AraC was kindly provided by the pharmacy of the TUH. For control,

$447 \quad$ NSG mice were treated daily with intraperitoneal injection of vehicle, PBS 1X.

448 - IACS-10759 treatment: IACS-10759 was solubilized in water containing $0.5 \%$

449 methylcellulose before administration to mice. NSG mice were treated 3 times a week by 
450 gavage of 8 or $5 \mathrm{mg} / \mathrm{kg}$ IACS-10759 (according to weight loss of mice) for 21 days. For

451 control, NSG mice were treated by daily gavage of vehicle. IACS-10759 was kindly

452 provided by Dr. Joe Marszalek.

453 - AG-120 treatment: AG-120 (Ivosidenib, AGIOS Pharmaceuticals) was solubilized in water 454 containing $0.5 \%$ methylcellulose and $0.2 \%$ Tween 80 before administration to mice. NSG 455 mice were treated twice a day by gavage of $150 \mathrm{mg} / \mathrm{kg}$ AG-120 for 21 days. For control, $456 \quad$ NSG mice were treated twice a day by gavage of vehicle.

457 Mice were monitored for toxicity and provided nutritional supplements as needed.

458 Assessment of Leukemic Engraftment

459 Assessment of leukemic engraftment was measured as reported previously ${ }^{11}$. Briefly, NSG 460 mice were humanely killed in accordance with European ethics protocols. Bone marrow 461 (mixed from tibias and femurs) and spleen were dissected in a sterile environment and flushed 462 in Hank's Balanced Salt Solution with 1\% FBS. MNCs from peripheral blood, bone marrow, 463 and spleen were labeled with hCD33-PE (555450), mCD45.1-PerCP-Cy5.5 (156058), 464 hCD45-APC (5555485), and hCD44-PECy (7560533) (all antibodies from BD Biosciences) 465 to determine the fraction of human blasts (hCD45 $5^{+} \mathrm{mCD} 45.1{ }^{-h C D} 33^{+} \mathrm{hCD} 44^{+}$cells) using 466 flow cytometry. All antibodies used for cytometry were used at concentrations between 1/50 467 and 1/200 depending on specificity and cell density. Analyses were performed on a 468 CytoFLEX flow cytometer with CytoExpert software (Beckman Coulter) and FlowJo 10.2 469 (Tree Star). The number of AML cells/ $\mu$ l peripheral blood and number of AML cells in total 470 cell tumor burden (in bone marrow and spleen) were determined by using CountBright beads 471 (Invitrogen) using described protocol.

\section{Statistical analyses}

473 Statistical analyses were conducted using Prism software v6.0 (GraphPad Software, La Jolla, 474 CA, USA). For in vitro and in vivo studies, statistical significance was determined by the two- 
475 tailed unpaired Student's t-test. For transcriptomic analysis of cohorts, statistical significance

476 was determined by the non-parametric Mann-Withney test. A pvalue $<0.05$ was considered 477 statistically significant. For all figures, ns, not significant, ${ }^{*} \mathrm{p} \% 0.05,{ }^{*} \mathrm{p} \% 00.01,{ }^{* * *} \mathrm{p} \% 0.001$, $478 * * * * \mathrm{p} \% 0.0001$. Unless otherwise indicated, all data represent the mean \pm standard error of the 479 mean (SEM) from at least three independent experiments. Box-and-whisker plots displays all 480 the individual data points as well as the corresponding median. For metabolomic analysis, 481 Seahorse and ATP assays, each biological replicates represents the mean of at least two 482 technical replicates.

483

484

Data availability statement

RNAseq data from Fig. 3 are part of a clinical trial and available upon request.

487 Acknowledgements

488 We thank The Cancéropoles PACA and GSO, the Network MetaboCancerGSO, Karine 489 Marendziak, all members of mice core facilities (UMS006, ANEXPLO, Inserm, Toulouse) 490 for their support and technical assistance, and Audrey Sarry, Prof. Véronique De Mas and 491 Eric Delabesse for the management of the Biobank BRC-HIMIP (Biological Resources 492 Centres-Inserm Midi-Pyrénées “Cytothèque des hémopathies malignes”) that is supported by 493 CAPTOR (Cancer Pharmacology of Toulouse-Oncopole and Région). We thank Anne-Marie 494 Benot, Muriel Serthelon and Stéphanie Nevouet for their daily help about the administrative 495 and financial management of the Sarry lab. We thank the Institut Paoli Calmettes Direction de 496 la Recherche Clinique et de l'Innovation (DRCI), the Hematology Department, and the 497 IPC/CRCM Biological Resource Center for sample collection and processing. We also thank 498 patients and their families.

499 This work was also supported by grants from the Région Midi-Pyrénées (FLEXAML, J-E.S.), 
500 the Plan Cancer Biologie des Systèmes 2014 (FLEXAML; J-E.S.), the Laboratoire

501 d'Excellence Toulouse Cancer (TOUCAN; contract ANR11-LABEX), the Canceropole GSO

502 (MetaboCancerGSO; J-E.S., J-C.P., L.L.C.) the Programme Hospitalo-Universitaire en

503 Cancérologie (CAPTOR; contract ANR11-PHUC0001), La Ligue Nationale de Lutte Contre

504 le Cancer, the Fondation ARC, the Fondation Toulouse Cancer Sante and the Association

505 G.A.E.L. MetaTOUL (Metabolomics \& Fluxomics Facitilies, Toulouse, France,

506 www.metatoul.fr) and LEMM are part of the national infrastructure MetaboHUB-ANR-11-

507 INBS-0010 (The French National infrastructure for metabolomics and fluxomics,

508 www.metabohub.fr). MetaToul is supported by grants from the Région Midi-Pyrénées, the

509 European Regional Development Fund, the SICOVAL, the Infrastructures en Biologie Santé

510 et Agronomie (IBiSa, France), the Centre National de la Recherche Scientifique (CNRS) and

511 the Institut National de la Recherche Agronomique (INRA). The project has been partly

512 supported by Canceroprole PACA, SIRIC (grant INCa-Inserm-DGOS 6038 2012-

513 2017), Canceropôle-SIRIC EmA, INCA 2017-024-COLLETTE and INCa 2017-024-MAL. C.

514 M. was supported by SIRIC (grant INCa-Inserm-DGOS 6038 2012-2017) and Canceropôle-

515 SIRIC EmA. E. T. was supported by SIRIC-Montpellier Cancer Program (grant INCa-Inserm-

516 DGOS 6045 2012-2017). N. Ba. and M. K. were supported by funding from Leukemia Spore

517 grant P50 (CA100632-16).

518

519 Author contributions

520 L.S., M.S. and J-E.S designed experiments. L.S., M.S., C.B., E.S., T.F., N.Br., C.L., N. Ba.,

521 M. Co., S.L., C.Cas., G.Co., A.Z., M.H., H.B., L.K.L. performed in vitro experiments. M.S.,

522 C.B., E.S., E.B., T.F., P-L.M, M.G. performed in vivo experiments. C.M. and R.C. performed

523 chemogrammes' analysis. L.S., F.C., L.G., E.T., E.C-V, A.T. and G. Ca. performed

524 metabolomics analyses. N.P., L.F. and F.J. performed genome-scale metabolic network 
525 analysis. L.S., T.F. and T.K. performed transcriptomic analysis on publically available

526 cohorts. F.W., K.M., A.F. and K.T. performed and analyzed RNAseq analysis on patient

527 samples treated with IDHm inhibitors. AML bone marrow and blood samples were provided

528 by M.Ca. (UPENN), N.V. and Y.C. (IPC), C.Cal., A.P. and A.B. (BUH), P.B. and C.R.

529 (TUH), C.D.D and M.K. (MDACC). J. M. and M.K provided IACS-010759. B.N. and S.R.

530 provided and measured AG-120 in mice sera. L.L.C., F.F., A.T., Y.G., J.M., C.D.D, M.K.,

531 L.K.L, F.B. and J-C.P managed the resources and shared their expertise. L.S. and J-E.S. wrote

532 the manuscript and designed the figures. N.P., M.A.S., F.F., F.J., C.R. and J-C.P reviewed the

533 manuscript. J-E-S directed the research.

534

\section{Competing interests statement}

536 C.D.D. is a consultant for Agios and Celgene, and served on the advisory board for Bayer,

537 Karyopharm, MedImmune, and AbbVie. S.R. and B.N. are both employees and own stock in

538 Agios Pharmaceuticals.

539 The rest of the authors declare no competing interests.

540

\section{References}

542 1. Boroughs, L. K. \& DeBerardinis, R. J. Metabolic pathways promoting cancer cell 543 survival and growth. Nat. Cell Biol. 17, 351-359 (2015).

544 2. Lehuédé, C., Dupuy, F., Rabinovitch, R., Jones, R. G. \& Siegel, P. M. Metabolic

545 Plasticity as a Determinant of Tumor Growth and Metastasis. Cancer Res. 76, 5201-8

$546 \quad$ (2016).

547 3. Martinez-Outschoorn, U. E., Peiris-Pagés, M., Pestell, R. G., Sotgia, F. \& Lisanti, M.

548 P. Cancer metabolism: a therapeutic perspective. Nat. Rev. Clin. Oncol. 14, 11-31 $549 \quad$ (2017).

550 4. Vander Heiden, M. G. \& DeBerardinis, R. J. Understanding the Intersections between $551 \quad$ Metabolism and Cancer Biology. Cell 168, 657-669 (2017).

552 5. Samudio, I. et al. Pharmacologic inhibition of fatty acid oxidation sensitizes human 553 leukemia cells to apoptosis induction. J. Clin. Invest. 120, 142-56 (2010).

554 6. Škrtić, M. et al. Inhibition of Mitochondrial Translation as a Therapeutic Strategy for $555 \quad$ Human Acute Myeloid Leukemia. Cancer Cell 20, 674-688 (2011).

556 7. Scotland, S. et al. Mitochondrial energetic and AKT status mediate metabolic effects 557 and apoptosis of metformin in human leukemic cells. Leukemia 27, 2129-2138 (2013).

558 8. Jacque, N. et al. Targeting glutaminolysis has antileukemic activity in acute myeloid 
602

603

604

605

606

607

608

leukemia and synergizes with BCL-2 inhibition. Blood 126, 1346-56 (2015).

9. Matre, P. et al. Inhibiting glutaminase in acute myeloid leukemia: metabolic dependency of selected AML subtypes. Oncotarget 7, 79722-79735 (2016).

10. Poulain, L. et al. High mTORC1 activity drives glycolysis addiction and sensitivity to G6PD inhibition in acute myeloid leukemia cells. Leukemia 31, 2326-2335 (2017).

11. Farge, T. et al. Chemotherapy-Resistant Human Acute Myeloid Leukemia Cells Are Not Enriched for Leukemic Stem Cells but Require Oxidative Metabolism. Cancer Discov. 7, 716-735 (2017).

12. Bosc, C., Selak, M. A. \& Sarry, J.-E. Resistance Is Futile: Targeting Mitochondrial Energetics and Metabolism to Overcome Drug Resistance in Cancer Treatment. Cell Metab. 26, 705-707 (2017).

13. Stuani, L., Sabatier, M. \& Sarry, J.-E. Exploiting metabolic vulnerabilities for personalized therapy in acute myeloid leukemia. BMC Biol. 17, 57 (2019).

14. Mardis, E. R. et al. Recurring Mutations Found by Sequencing an Acute Myeloid Leukemia Genome. N. Engl. J. Med. 361, 1058-1066 (2009).

15. Abbas, S. et al. Acquired mutations in the genes encoding IDH1 and IDH2 both are recurrent aberrations in acute myeloid leukemia: prevalence and prognostic value. Blood 116, 2122-6 (2010).

16. Marcucci, G. et al. IDH1 and IDH2 gene mutations identify novel molecular subsets within de novo cytogenetically normal acute myeloid leukemia: a Cancer and Leukemia Group B study. J. Clin. Oncol. 28, 2348-55 (2010).

17. Paschka, P. et al. IDH1 and IDH2 mutations are frequent genetic alterations in acute myeloid leukemia and confer adverse prognosis in cytogenetically normal acute myeloid leukemia with NPM1 mutation without FLT3 internal tandem duplication. $J$. Clin. Oncol. 28, 3636-43 (2010).

18. Figueroa, M. E. et al. Leukemic IDH1 and IDH2 Mutations Result in a Hypermethylation Phenotype, Disrupt TET2 Function, and Impair Hematopoietic Differentiation. Cancer Cell 18, 553-567 (2010).

19. Sasaki, M. et al. D-2-hydroxyglutarate produced by mutant IDH1 perturbs collagen maturation and basement membrane function. Genes Dev. 26, 2038-49 (2012).

20. Konopleva, M. et al. Efficacy and Biological Correlates of Response in a Phase II Study of Venetoclax Monotherapy in Patients with Acute Myelogenous Leukemia. Cancer Discov. 6, 1106-1117 (2016).

21. Losman, J.-A. et al. (R)-2-hydroxyglutarate is sufficient to promote leukemogenesis and its effects are reversible. Science 339, 1621-5 (2013).

22. Kats, L. M. et al. Proto-oncogenic role of mutant IDH2 in leukemia initiation and maintenance. Cell Stem Cell 14, 329-41 (2014).

23. Boutzen, H. et al. Isocitrate dehydrogenase 1 mutations prime the all-trans retinoic acid myeloid differentiation pathway in acute myeloid leukemia. J. Exp. Med. 213, 483-97 (2016).

24. Inoue, S. et al. Mutant IDH1 Downregulates ATM and Alters DNA Repair and Sensitivity to DNA Damage Independent of TET2. Cancer Cell 30, 337-348 (2016).

25. Elkashef, S. M. et al. IDH Mutation, Competitive Inhibition of FTO, and RNA Methylation. Cancer Cell 31, 619-620 (2017).

26. Jiang, B. et al. IDH1 Mutation Promotes Tumorigenesis by Inhibiting JNK Activation and Apoptosis Induced by Serum Starvation. Cell Rep. 19, 389-400 (2017).

27. Turcan, S. et al. Mutant-IDH1-dependent chromatin state reprogramming, reversibility, and persistence. Nat. Genet. 50, 62-72 (2018).

28. Chan, S. M. et al. Isocitrate dehydrogenase 1 and 2 mutations induce BCL-2 dependence in acute myeloid leukemia. Nat. Med. 21, 178-184 (2015). 
29. Corces-Zimmerman, M. R. \& Majeti, R. Pre-leukemic evolution of hematopoietic stem cells: the importance of early mutations in leukemogenesis. Leukemia 28, 2276-2282 (2014).

30. Shlush, L. I. et al. Identification of pre-leukaemic haematopoietic stem cells in acute leukaemia. Nature 506, 328-333 (2014).

31. Ok, C. Y. et al. Persistent IDH1/2 mutations in remission can predict relapse in patients with acute myeloid leukemia. Haematologica 104, 305-311 (2019).

32. Popovici-Muller, J. et al. Discovery of the First Potent Inhibitors of Mutant IDH1 That Lower Tumor 2-HG in Vivo. ACS Med. Chem. Lett. 3, 850-855 (2012).

33. Rohle, D. et al. An inhibitor of mutant IDH1 delays growth and promotes differentiation of glioma cells. Science 340, 626-30 (2013).

34. Wang, F. et al. Targeted inhibition of mutant IDH2 in leukemia cells induces cellular differentiation. Science 340, 622-6 (2013).

35. Okoye-Okafor, U. C. et al. New IDH1 mutant inhibitors for treatment of acute myeloid leukemia. Nat. Chem. Biol. 11, 878-886 (2015).

36. Yen, K. et al. AG-221, a First-in-Class Therapy Targeting Acute Myeloid Leukemia Harboring Oncogenic IDH2 Mutations. Cancer Discov. 7, 478-493 (2017).

37. Stein, E. M. et al. Enasidenib in mutant IDH2 relapsed or refractory acute myeloid leukemia. Blood 130, 722-731 (2017).

38. DiNardo, C. D. et al. Durable Remissions with Ivosidenib in IDH1 -Mutated Relapsed or Refractory AML. N. Engl. J. Med. NEJMoa1716984 (2018). doi:10.1056/NEJMoa1716984

39. Pollyea, D. A. et al. Enasidenib, an inhibitor of mutant IDH2 proteins, induces durable remissions in older patients with newly diagnosed acute myeloid leukemia. Leukemia 1 (2019). doi:10.1038/s41375-019-0472-2

40. Stein, E. M. et al. Molecular remission and response patterns in patients with mutantIDH2 acute myeloid leukemia treated with enasidenib. Blood 133, 676-687 (2019).

41. DiNardo, C. D. \& Perl, A. E. Advances in patient care through increasingly individualized therapy. Nat. Rev. Clin. Oncol. 16, 73-74 (2019).

42. DiNardo, C. et al. Molecular Profiling and Relationship with Clinical Response in Patients with IDH1 Mutation-Positive Hematologic Malignancies Receiving AG-120, a First-in-Class Potent Inhibitor of Mutant IDH1, in Addition to Data from the Completed Dose Escalation Portio.... Blood 126, (2015).

43. De Botton, S., Pollyea, D. A. \& Stein, E. M. Clinical safety and activity of AG-120, a first-in-class, potent inhibitor of the IDH1 mutant protein, in a phase 1 study of patients with advanced IDH1-mutant hematologic malignancies [abstract]. Haematologica 100, 214-215 (2015).

44. Amatangelo, M. D. et al. Enasidenib induces acute myeloid leukemia cell differentiation to promote clinical response. Blood 130, 732-741 (2017).

45. Thomas, D. \& Majeti, R. Optimizing Next-Generation AML Therapy: Activity of Mutant IDH2 Inhibitor AG-221 in Preclinical Models. Cancer Discov. 7, 459-461 (2017).

46. Waitkus, M. S., Diplas, B. H. \& Yan, H. Biological Role and Therapeutic Potential of IDH Mutations in Cancer. Cancer Cell (2018). doi:10.1016/J.CCELL.2018.04.011

47. Jones, C. L. et al. Cysteine depletion targets leukemia stem cells through inhibition of electron transport complex II. Blood blood.2019898114 (2019). doi:10.1182/BLOOD.2019898114

48. Intlekofer, A. M. et al. Acquired resistance to IDH inhibition through trans or cis dimer-interface mutations. Nature 559, 125-129 (2018).

49. Quek, L. et al. Clonal heterogeneity of acute myeloid leukemia treated with the IDH2 
659

660

661

662

663

664

665

666

667

668

669

670

671

672

673

674

675

676

677

678

679

680

681

682

683

684

685

686

687

688

689

690

691

692

693

694

695

696

697

698

699

700

701

702

703

704

705

706

707

708

inhibitor enasidenib. Nat. Med. 1 (2018). doi:10.1038/s41591-018-0115-6

50. Harding, J. J. et al. Isoform Switching as a Mechanism of Acquired Resistance to Mutant Isocitrate Dehydrogenase Inhibition. Cancer Discov. 8, 1540-1547 (2018).

51. Tommasini-Ghelfi, S. et al. Cancer-associated mutation and beyond: The emerging biology of isocitrate dehydrogenases in human disease. Sci. $A d v$. 5, eaaw4543 (2019).

52. Tateishi, K. et al. Extreme Vulnerability of IDH1 Mutant Cancers to NAD+ Depletion. Cancer Cell 28, 773-784 (2015).

53. Fack, F. et al. Altered metabolic landscape in IDH-mutant gliomas affects phospholipid, energy, and oxidative stress pathways. EMBO Mol. Med. 9, 1681-1695 (2017).

54. Maus, A. \& Peters, G. J. Glutamate and $\alpha$-ketoglutarate: key players in glioma metabolism. Amino Acids 49, 21-32 (2017).

55. Hollinshead, K. E. R. et al. Oncogenic IDH1 Mutations Promote Enhanced Proline Synthesis through PYCR1 to Support the Maintenance of Mitochondrial Redox Homeostasis. Cell Rep. 22, 3107-3114 (2018).

56. Gelman, S. J. et al. Consumption of NADPH for 2-HG Synthesis Increases Pentose Phosphate Pathway Flux and Sensitizes Cells to Oxidative Stress. Cell Rep. 22, 512 522 (2018).

57. Zhou, L. et al. Integrated Metabolomics and Lipidomics Analyses Reveal Metabolic Reprogramming in Human Glioma with IDH1 Mutation. (2018). doi:10.1021/acs.jproteome. 8 b00663

58. Badur, M. G. et al. Oncogenic R132 IDH1 Mutations Limit NADPH for De Novo Lipogenesis through (D)2-Hydroxyglutarate Production in Fibrosarcoma Cells. Cell Rep. 25, 1018-1026.e4 (2018).

59. Grassian, A. R. et al. IDH1 mutations alter citric acid cycle metabolism and increase dependence on oxidative mitochondrial metabolism. Cancer Res. 74, 3317-31 (2014).

60. Izquierdo-Garcia, J. L. et al. Glioma Cells with the IDH1 Mutation Modulate Metabolic Fractional Flux through Pyruvate Carboxylase. PLoS One 9, e108289 (2014).

61. Cuyàs, E. et al. Oncometabolic mutation IDH1 R132H confers a metforminhypersensitive phenotype. Oncotarget 6, 12279-12296 (2015).

62. Chan, S. M. et al. Isocitrate dehydrogenase 1 and 2 mutations induce BCL-2 dependence in acute myeloid leukemia. Nat. Med. 21, 178-184 (2015).

63. Molina, J. R. et al. An inhibitor of oxidative phosphorylation exploits cancer vulnerability. Nat. Med. 24, 1036-1046 (2018).

64. Kuntz, E. M. et al. Targeting mitochondrial oxidative phosphorylation eradicates therapy-resistant chronic myeloid leukemia stem cells. Nat. Med. 23, 1234-1240 (2017).

65. Lee, K.-M. et al. MYC and MCL1 Cooperatively Promote Chemotherapy-Resistant Breast Cancer Stem Cells via Regulation of Mitochondrial Oxidative Phosphorylation. Cell Metab. 26, 633-647.e7 (2017).

66. Thiele, I. et al. A community-driven global reconstruction of human metabolism. Nat. Biotechnol. 31, 419-425 (2013).

67. Jerby, L., Shlomi, T. \& Ruppin, E. Computational reconstruction of tissue-specific metabolic models: application to human liver metabolism. Mol. Syst. Biol. 6, (2010).

68. Poupin, N. et al. Large-Scale Modeling Approach Reveals Functional Metabolic Shifts during Hepatic Differentiation. J. Proteome Res. acs.jproteome.8b00524 (2018). doi:10.1021/acs.jproteome.8b00524

69. Verhaak, R. G. W. et al. Prediction of molecular subtypes in acute myeloid leukemia based on gene expression profiling. Haematologica 94, 131-4 (2009). 
70. Network, T. C. G. A. R. Genomic and Epigenomic Landscapes of Adult De Novo Acute Myeloid Leukemia. N. Engl. J. Med. 368, 2059-2074 (2013).

71. Lu, C. et al. IDH mutation impairs histone demethylation and results in a block to cell differentiation. Nature 483, 474-478 (2012).

72. Kernytsky, A. et al. IDH2 mutation-induced histone and DNA hypermethylation is progressively reversed by small-molecule inhibition. Blood 125, 296-303 (2015).

73. Viale, A. et al. Oncogene ablation-resistant pancreatic cancer cells depend on mitochondrial function. Nature 514, 628-632 (2014).

74. Lee, E. A. et al. Targeting Mitochondria with Avocatin B Induces Selective Leukemia Cell Death. Cancer Res. 75, 2478-2488 (2015).

75. Chou, A. P. et al. Identification of Retinol Binding Protein 1 Promoter Hypermethylation in Isocitrate Dehydrogenase 1 and 2 Mutant Gliomas. JNCI J. Natl. Cancer Inst. 104, 1458-1469 (2012).

76. Guilhamon, P. et al. Meta-analysis of IDH-mutant cancers identifies EBF1 as an interaction partner for TET2. Nat. Commun. 4, 2166 (2013).

77. Flavahan, W. A. et al. Insulator dysfunction and oncogene activation in IDH mutant gliomas. Nature 529, 110-114 (2016).

78. Mazor, T. et al. Clonal expansion and epigenetic reprogramming following deletion or amplification of mutant IDH1. Proc. Natl. Acad. Sci. U. S. A. 114, 10743-10748 (2017).

79. Farshidfar, F. et al. Integrative Genomic Analysis of Cholangiocarcinoma Identifies Distinct IDH-Mutant Molecular Profiles. Cell Rep. 19, 2878-2880 (2017).

80. Khurshed, M., Molenaar, R. J., Lenting, K., Leenders, W. P. \& van Noorden, C. J. F. In silico gene expression analysis reveals glycolysis and acetate anaplerosis in IDH1 wildtype glioma and lactate and glutamate anaplerosis in IDH1-mutated glioma. Oncotarget 8, 49165-49177 (2017).

81. Sriskanthadevan, S. et al. AML cells have low spare reserve capacity in their respiratory chain that renders them susceptible to oxidative metabolic stress. Blood 125, 2120-30 (2015).

82. Baccelli, I. et al. Mubritinib Targets the Electron Transport Chain Complex I and Reveals the Landscape of OXPHOS Dependency in Acute Myeloid Leukemia. Cancer Cell 36, 84-99.e8 (2019).

83. Bisaillon, R. et al. Genetic characterization of ABT-199 sensitivity in human AML. Leukemia 1 (2019). doi:10.1038/s41375-019-0485-x

84. Wouters, B. J. Hitting the target in IDH2 mutant AML. Blood 130, 693-694 (2017).

85. Ye, D., Guan, K.-L. \& Xiong, Y. Metabolism, Activity, and Targeting of D- and L-2Hydroxyglutarates. Trends in cancer 4, 151-165 (2018).

86. Oizel, K. et al. D-2-Hydroxyglutarate does not mimic all the IDH mutation effects, in particular the reduced etoposide-triggered apoptosis mediated by an alteration in mitochondrial NADH. Cell Death Dis. 6, e1704 (2015).

87. Nussinov, R., Tsai, C.-J. \& Jang, H. Protein ensembles link genotype to phenotype. PLOS Comput. Biol. 15, e1006648 (2019).

88. Letai, A. Functional precision cancer medicine-moving beyond pure genomics. Nat. Med. 23, 1028-1035 (2017).

89. Saland, E. et al. A robust and rapid xenograft model to assess efficacy of chemotherapeutic agents for human acute myeloid leukemia. Blood Cancer J. 5, e297 (2015).

90. Sanchez, P. V et al. A robust xenotransplantation model for acute myeloid leukemia. Leukemia 23, 2109-2117 (2009).

91. Sarry, J.-E. et al. Human acute myelogenous leukemia stem cells are rare and 
764

765

766

767

768

769

770

771

772

773

774

775

776

777

778

779

780

781

782

783

784

785

786

787

788

789

790

791

792

793

794

heterogeneous when assayed in NOD/SCID/IL2R $\gamma \mathrm{c}$-deficient mice. J. Clin. Invest. 121, 384-95 (2011).

92. Mookerjee, S. A., Gerencser, A. A., Nicholls, D. G. \& Brand, M. D. Quantifying intracellular rates of glycolytic and oxidative ATP production and consumption using extracellular flux measurements. J. Biol. Chem. 292, 7189-7207 (2017).

93. Zur, H., Ruppin, E. \& Shlomi, T. iMAT: an integrative metabolic analysis tool. Bioinformatics 26, 3140-3142 (2010).

94. Chazalviel, M. et al. MetExploreViz: web component for interactive metabolic network visualization. Bioinformatics 34, 312-313 (2018).

95. Cottret, L. et al. MetExplore: a web server to link metabolomic experiments and genome-scale metabolic networks. Nucleic Acids Res. 38, W132-W137 (2010).

96. Tyner, J. W. et al. Functional genomic landscape of acute myeloid leukaemia. Nature 562, 526-531 (2018).

97. Subramanian, A. et al. Gene set enrichment analysis: a knowledge-based approach for interpreting genome-wide expression profiles. Proc. Natl. Acad. Sci. U. S. A. 102, 15545-50 (2005).

98. Mootha, V. K. et al. PGC-1 $\alpha$-responsive genes involved in oxidative phosphorylation are coordinately downregulated in human diabetes. Nat. Genet. 34, 267-273 (2003).

\section{Figure legends}

Figure 1. IDH1 mutant cells exhibit a higher susceptibility to $O x P H O S_{i}$ and $B C L 2_{i}$ is linked to their enhanced mitochondrial capabilities and OxPHOS activity in AML.

(a) Schematic representation of the electron transport chain (ETC) and BCL2 with OXPHOSi and BCL2 $\mathrm{i}$ used in this study (Metf= metformin; $\mathrm{AA}=$ antimycin $\mathrm{A} ; \mathrm{ATVQ}=$ atovaquone; oligo=oligomycin)

(b) Plots of EC50 values from ATP viability assays of metformin and ABT-263 after 48h, from annexin V positive cells assays of ABT-199 after 24h and percent of viable cells after $72 \mathrm{~h}$ of IACS-010759 in primary samples with WT or MUT IDH1 (red circles) or IDH2 (burgundy circles). See also Supplementary Table 1 for patient information.

(c) Apoptosis induction following IACS-010759 (100nM during 48h for HL60 and during 6 days for MOLM14), 48h metformin $(10 \mathrm{mM})$, antimicyn A $(10 \mu \mathrm{M})$, atovaquone $(20 \mu \mathrm{M}$ for

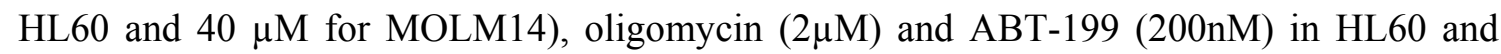
MOLM14 IDH1 WT or R132H. Errors bars indicate Mean \pm SEM of at least three independent experiments. 
795 (d) $2-\mathrm{HG}$ fold change following $24 \mathrm{~h}$ treatment with metformin (10mM) and ABT-199 796 (200nM) in HL60 and MOLM14 IDH1 WT or R132H. Errors bars indicate Mean \pm SEM of at 797 least two independent experiments.

798 (e)TMRE mitochondrial potential assay in HL60 and MOLM14 IDH1 WT or R132H 799 measured in vitro ( $\mathrm{n} \geq 3$ ) and in vivo in PDX (3 patients IDH1 WT and 3 patients IDH1 MUT) 800 to estimate Mitochondrial Membrane Potential (MMP). See also Supplementary Table 1 for 801 patient information. Errors bars indicate Mean \pm SEM.

802 (f) Mitochondrial Oxygen Consumption Rate (OCR) of HL60 and MOLM14 IDH1 WT or $803 \mathrm{R} 132 \mathrm{H}$ measured in vitro $(\mathrm{n} \geq 3)$ and ex vivo in PDX after cell-sorting (4 patients IDH1 WT 804 and 2 patients IDH1 MUT). See also Supplementary Table 1 for patient information. Errors 805 bars indicate Mean \pm SEM.

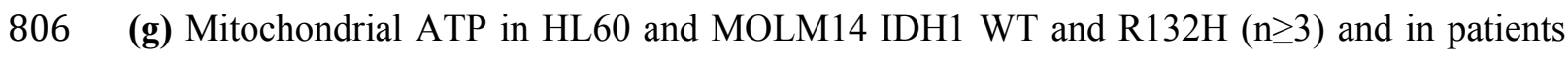
807 with IDH WT ( $\mathrm{n}=14)$ or MUT IDH1 (red circles) or IDH2 (burgundy circles) ( $\mathrm{n}=21)$. See also 808 Supplementary Table 1 for patient information. Errors bars indicate Mean \pm SEM.

809 (h) Mitochondrial ETC complex activities in HL60 and MOLM14 IDH1 WT and R132H. 810 Errors bars indicate Mean \pm SEM of at least three independent experiments.

811 (i) NADH-producing enzyme activities of malate dehydrogenase (MDH) and isocitrate 812 dehydrogenase (IDH3) in HL60 and MOLM14 IDH1 WT and R132H. Errors bars indicate 813 Mean \pm SEM of at least three independent experiments.

814 (j) Succinate (succ), fumarate (fum), malate (mal), cis-aconitate (cis-aco), citrate (cit) and $\alpha$ $815 \mathrm{KG}$ amounts measured over 24h culture in HL60 and MOLM14 IDH1 WT and R132H. 816 Errors bars indicate Mean $\pm \mathrm{SEM}$ of at least three independent experiments.

817 (k) Plots of $\mathrm{EC}_{50}$ values of AraC determined from ATP viability assays at $48 \mathrm{~h}$ (left panel) and 818 total number of human viable AML cells expressing CD45 and CD33 in AraC-treated 
819 compared with PBS-treated IDH1 mutant AML-xenografted mice in bone marrow and spleen

820 (right panel). See also Supplementary Table 1 for patient information.

821 For each panel (b-k), HL60 IDH1 WT are represented in blue by circles (clone 4), triangles

822 up (clone 2) and triangles down (clone 7) whereas $\mathrm{R} 132 \mathrm{H}$ are represented in red by circles

823 (clone 11) and triangles up (clone 5). MOLM14 are represented by squares, blue for IDH1

824 WT and red for IDH1 R132H (both induced by doxycycline). Groups were compared with

825 unpaired two-tailed t test with Welch's correction. ${ }^{*} \mathrm{p}<0.05 ;{ }^{*} \mathrm{p}<0.01 ; * * * \mathrm{p}<0.001$; ns, not 826 significant.

827

828 Figure 2. Methylation- and CEBP $\alpha$ - dependent mitochondrial fatty acid oxidation is

829 increased in IDH1 mutant cells.

830 (a) Comparison of the predicted activity of reactions in the metabolic network of HL60 IDH1

831 WT vs. R132H cells. Predictions of reactions activity or inactivity was made using the Recon

8322 metabolic network reconstruction and transcriptomic data from HL60 IDH1 WT and

$833 \mathrm{R} 123 \mathrm{H}^{23}$. Pathway enrichment was performed on the set of reactions identified as specifically

834 active (red) or specifically inactive (blue) in $\mathrm{R} 123 \mathrm{H}$ cells. Corrected $p$-values were obtained

835 by performing a hypergeometric test followed by a Bonferroni correction.

836 (b) Visualization of modulated reactions within the fatty acid oxidation pathway of the Recon

8372 metabolic network. Reactions predicted to be specifically active (red) or inactive (blue) in

$838 \mathrm{R} 123 \mathrm{H}$ cells based on transcriptomic data alone (left panel) or using the computational

839 modeling approach (right panel) were mapped using the MetExplore webserver ${ }^{94,95}$.

840 (c) Acetyl-CoA, succinyl-CoA, free coenzyme A (CoASH) amounts measured over 24h

841 culture in HL60 IDH1 WT clone 4 and R132H clone 11 lysates. Errors bars indicate

842 Mean \pm SEM of two independent experiments with 2 technical replicates for each analyzed

843 with unpaired two-tailed t test with Welch's correction. 
844 (d) ${ }^{14} \mathrm{C}$ palmitate oxidation by HL60 IDH1 WT clone 4 (circle) and 2 (triangle) and R132H

845 clone 11 (circle) and 5 (triangle) to assess $\beta$-oxidation rate. Errors bars indicate Mean $\pm \mathrm{SEM}$

846 of six independent experiments analyzed with unpaired two-tailed t test with Welch's

847 correction.

848 (e) CPT1a gene expression across AML patient samples from GSE14468 (Verhaak cohort)

849 and BeatAML ${ }^{96}$ datasets in function of their IDH1 status. Groups were compared using

850 unpaired non-parametric Mann-Whitney test.

851 (f) Total lysates (left panel) and lysates of purified mitochondria (mito) (right panel) of HL60

852 and MOLM14 IDH1 WT and R132H and total lysates of primary samples IDH1 WT or MUT

853 (bottom left panel) were immunoblotted with the indicated antibodies. See also

854 Supplementary table 1 for patient information.

855 (g) Normalized enrichment score (NES) following GSEA analysis of patients with high or

856 low expression of CPT1a (mediane as the reference) in IDH WT, IDH1 mutant or IDH1+2

857 mutant across AML transcriptomes from two-independent cohorts, BeatAML and Verhaak

858 (GSE14468).

859 (h) qChIP experiments showing the relative recruitment of CEBP $\alpha$ on CPT1a, CPT2 and 860 SLC25A20 locus in mutant IDH1 R132H versus IDH1 WT HL60 and MOLM14, as indicated.

861 Results were represented as the relative ratio between the mean value of immunoprecipitated

862 chromatin (calculated as a percentage of the input) with the indicated antibodies and the one

863 obtained with a control irrelevant antibody. HL60 IDH1 WT are represented in blue by circles

864 (clone 4) and triangles (clone 2) whereas R132H are represented in red by circles (clone 11)

865 and triangles (clone 5). MOLM14 are represented by squares, blue for IDH1 WT and red for

866 IDH1 R132H (both induced by doxycycline). Errors bars indicate Mean \pm SEM of at least two

867 independent experiments analyzed with unpaired two-tailed t test with Welch's correction.

$868 * \mathrm{p}<0.05 ; * * \mathrm{p}<0.01 ; * * * \mathrm{p}<0.001 ; * * * * \mathrm{p}<0.0001 ;$ ns, not significant. 
Figure 3. IDH mutant inhibitors reverse 2 -HG production but do not necessarily 872 decrease high OxPHOS phenotype and mitochondrial metabolism.

873 (a) GSEA Normalized Enrichment Score (NES) from several transcriptomic signatures of 874 AML patients with IDH mutation characterized as responders to IDHm inhibitor before 875 treatment (8 patients), at complete remission CR (6 patients) and at relapse (5 patients); or as 876 non-responders to IDHm inhibitor (2 patients) before and after treatment with IDHm 877 inhibitor. See also Supplementary table 1 for patient information.

878 (b) Relative mRNA levels for CPT1a, CPT2, and SLC25A20 in AML patients with IDH 879 mutation characterized as responders to IDHm inhibitor before treatment ( 8 patients), at 880 complete remission CR (6 patients) and at relapse (5 patients).

881 (c) FAO-linked OCR in HL60 and MOLM14 IDH1 R132H following 1-week treatment with 882 AGI-5198 $(2 \mu \mathrm{M})$. Errors bars indicate Mean \pm SEM of four independent experiments.

883 (d) Mitochondrial OCR of HL60 and MOLM14 IDH1 R132H in vehicle (DMF) and after $88424 \mathrm{~h}, 1$ week or 2 weeks treatment with AGI-5198 $(2 \mu \mathrm{M})$. Errors bars indicate Mean \pm SEM of 885 at least three independent experiments.

886 (e) ATP-linked OCR of HL60 and MOLM14 IDH1 R132H in vehicle (DMF) and after 24h, 1 887 week or 2 weeks treatment with AGI-5198 $(2 \mu \mathrm{M})$. Errors bars indicate Mean \pm SEM of at least 888 three independent experiments.

889 (f) Citrate, succinate, malate and fumarate levels normalized to internal standard measured 890 over 24h culture in HL60 and MOLM14 IDH1 R132H following 24h, 1 week or 2 weeks

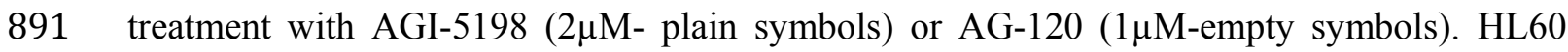
892 IDH1 R132H are represented in red by circles (clone 11) and triangles (clone 5), whereas 893 MOLM14 IDH1 R132H are represented by red squares. Errors bars indicate Mean \pm SEM of at 894 least three independent experiments. 
895 For panels (c-g), groups were compared with unpaired two-tailed t test with Welch's

896 correction. ${ }^{*} \mathrm{p}<0.05 ; * * \mathrm{p}<0.01 ; * * \mathrm{p}<0.001$; ns, not significant.

897

898

Figure 4. Treatment with an OxPHOS inhibitor enhances anti-leukemic effects of IDH-

mutant specific inhibitors alone and in combination with cytarabine in a high engrafter

900

IDH1 mutant AML PDX model.

901 (a) Experimental scheme detailing administration time of intraperitoneal AraC, IACS 010759

902 and AG-120 by gavages in PDX. See also Supplementary table 1 for patient information.

903 (b) AG-120 concentration in mice sera of PDX 325.

904

(c) 2-HG level normalized to control group in sera of IDH1 R132 PDX 325 mice after mono-,

905 duplet- or triplet-therapies compared with vehicle.

906 (d) Total number of human viable AML cells expressing CD45 and CD33 in mono-, duplet-

907 or triplet-therapies compared with vehicle and AraC-treated IDH1 R132 PDX 325 mice in

908 bone marrow and spleen. Fold change (FC) between each group and the mean of vehicle or

909 AraC group.

910 (e) Percent of human viable cells expressing CD15 in bone marrow in mono-, duplet- or

911 triplet-therapies compared with vehicle-treated IDH1 R132 PDX 325 mice.

912 (f) Percent of mitochondrial ATP contribution to total ATP after FACS-sorting of human

913 viable cells expressing CD45 and CD33 in bone marrow of IDH1 R132 PDX 325 mice

914 treated with mono-, duplet- or triplet-therapies compared with vehicle.

915 (g) Aspartate and lactate levels normalized to control group in mice sera of IDH1 R132 PDX 916325.

917 (h) Schematic diagram of metabolic reprogramming induced by IDH1 mutation in AML cells

918 and its impact on OxPHOS status through FAO regulation in absence of treatment, after

919 treatment with IDHm inhibitor then at relapse. 
bioRxiv preprint doi: https://doi.org/10.1101/749580; this version posted August 28,2019 . The copyright holder for this preprint (which was not certified by peer review) is the author/funder. All rights reserved. No reuse allowed without permission.

920 For panels (b-g), groups were compared with unpaired two-tailed t test with Welch's

921 correction. ${ }^{*} \mathrm{p}<0.05 ; * * \mathrm{p}<0.01 ; * * * \mathrm{p}<0.001 ; * * * * \mathrm{p}<0.0001 ;$ ns, not significant. 

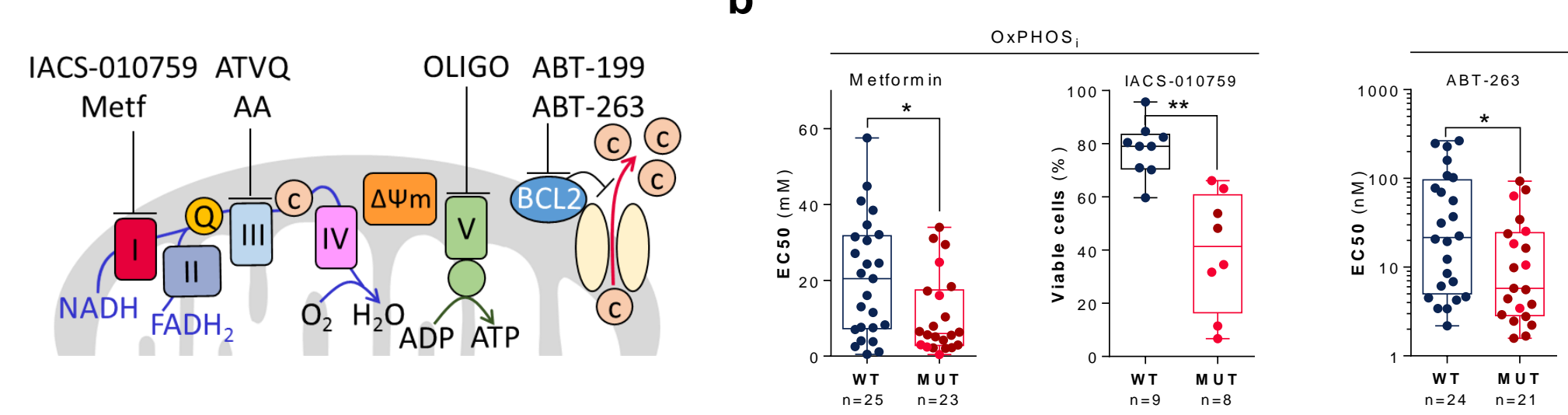

$n=24 \quad n=21$

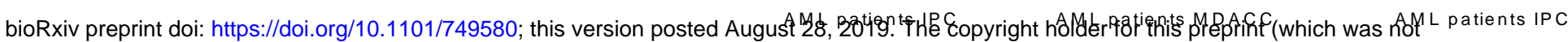
certified by peer review) is the author/funder. All rights reserved. No reuse allowed without permission.
C

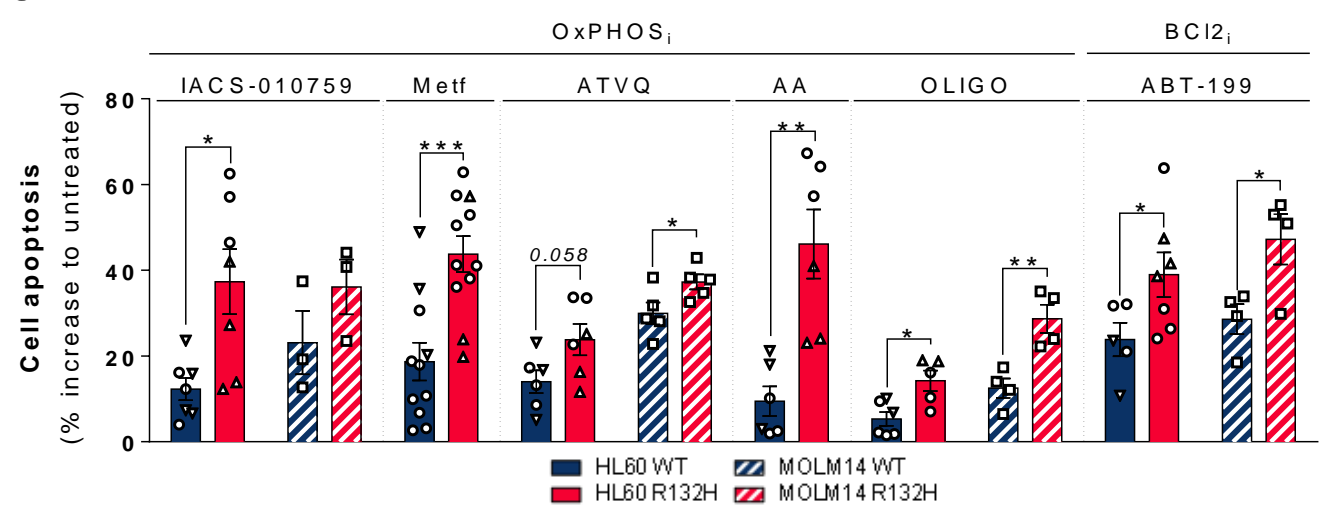

f

Mitochondrial OCR

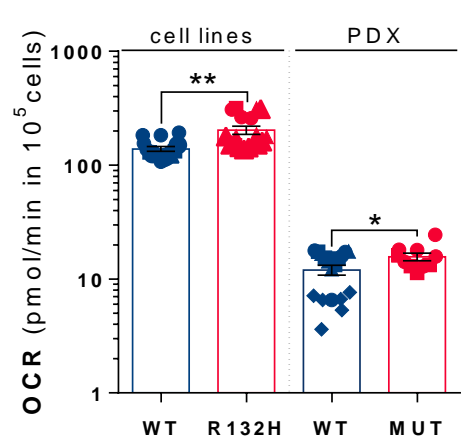

i

NADH-producing enzymes

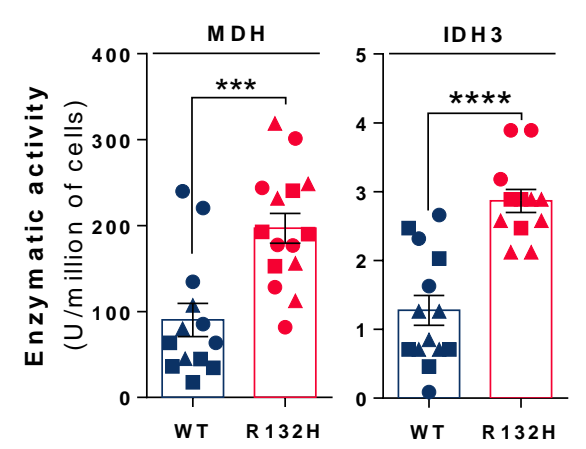

g

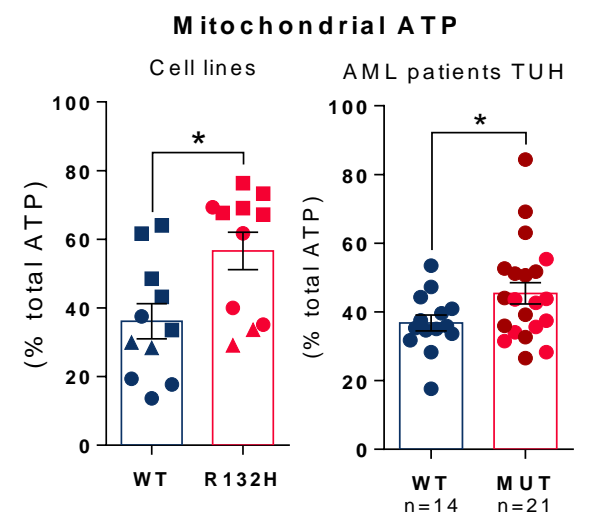

d

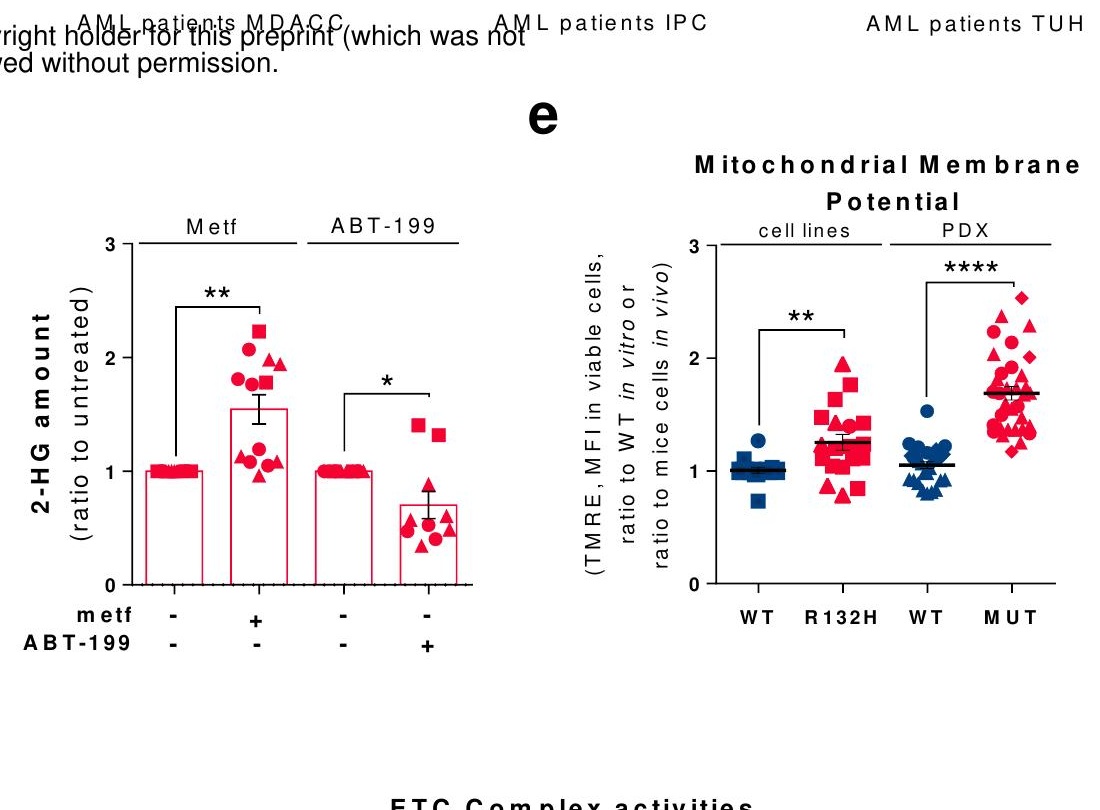

e

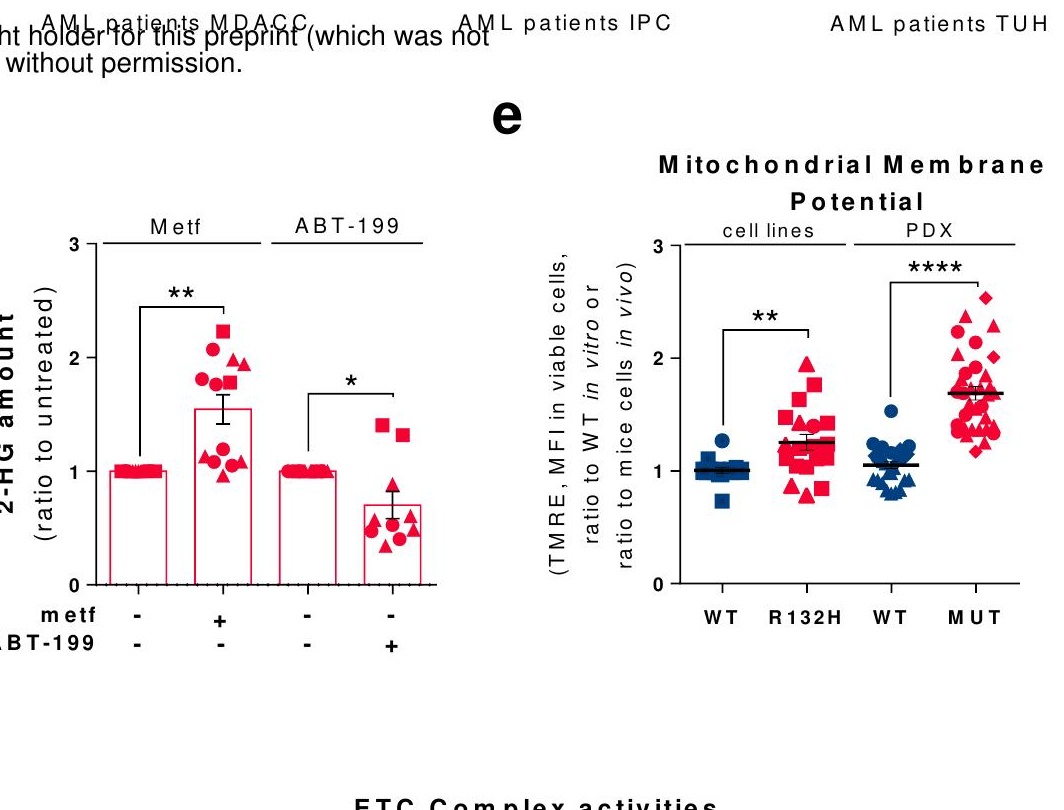

h

ETC Complex activities
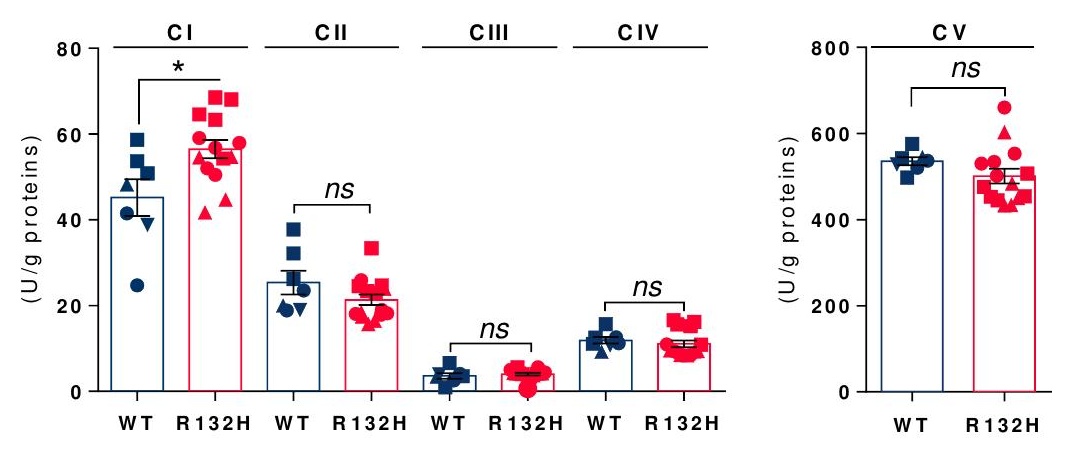

k

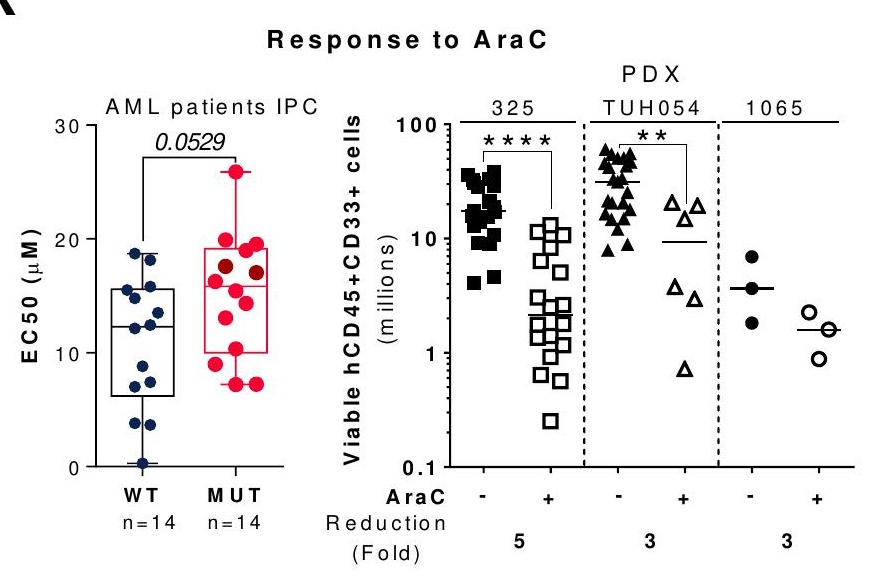




\begin{tabular}{|c|c|c|c|c|c|}
\hline \multirow{5}{*}{$\begin{array}{l}\text { Reactions } \\
\text { predicted } \\
\text { ACTIVE }\end{array}$} & \multirow{5}{*}{$\begin{array}{l}\text { reactions specifically } \\
\text { ACTIVE in R132H cells } \\
\qquad \begin{array}{l}(124)\end{array}\end{array}$} & Enriched pathways & $\begin{array}{l}\text { Nb reactions } \\
\text { mappedt/total } \\
\text { in pathway }\end{array}$ & $\begin{array}{c}\text { Corrected } \\
p \text {-value }\end{array}$ & Associated genes \\
\hline & & Fatty Acid oxidation & $84 / 577$ & $1.67 \mathrm{e}-43$ & CPT2; ACSL1; SLC25A20; CPT1A;CPT1C \\
\hline & & N-glycan synthesis & $16 / 83$ & $1.02 \mathrm{e}-08$ & MAN1A1; MAN1B1; MAN1C1 \\
\hline & & Fructose \& mannose metab. & $8 / 23$ & $1.93 \mathrm{e}-06$ & ALDOA; HK1; HK2; HK3; HDC1; ALDOC \\
\hline & & $\begin{array}{l}\text { Glyoxylate \& dicarboxylate } \\
\text { metab. }\end{array}$ & $4 / 11$ & $3.25 \mathrm{e}-03$ & 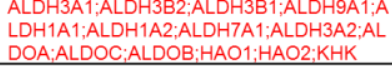 \\
\hline \multirow{3}{*}{$\begin{array}{l}\text { Reactions } \\
\text { predicted } \\
\text { INACTIVE }\end{array}$} & \multirow{3}{*}{$\begin{array}{l}\text { reactions specifically } \\
\text { INACTIVE in R132H cells } \\
\text { (148) }\end{array}$} & Fatty Acid oxidation & $71 / 577$ & $1.11 e-36$ & $\begin{array}{l}\text { LIPC; ACOT2; DBI; ECI1; DECR1; HADH; } \\
\text { ACAA2; ACADM }\end{array}$ \\
\hline & & Steroid metabolism & $6 / 50$ & $1.87 \mathrm{e}-02$ & $\begin{array}{l}\text { SRD5A1;:SRDSA2;AKR1C } \\
\text { UGT2B28 }\end{array}$ \\
\hline & & $\begin{array}{l}\text { Glyoxylate \& dicarboxylate } \\
\text { metab. }\end{array}$ & $3 / 11$ & $3.48 \mathrm{e}-02$ & $\begin{array}{l}\text { LDHAL6B;LDHC;LDHB;LDHA;LDHALE } \\
\text { DHD;GRHPR }\end{array}$ \\
\hline
\end{tabular}

Reaction activity status based on transcriptomic data and modelling mitochondria

mito/cyto Interface CPrepriat W which C

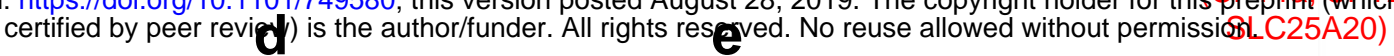

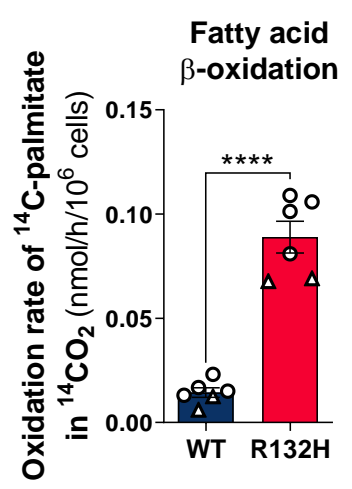

AML patients

HL60 MOLM14 WT R132H WT

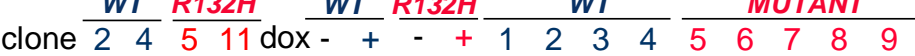

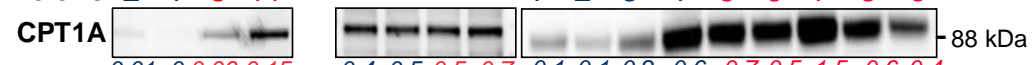

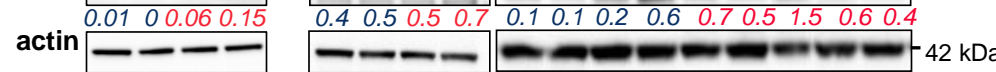

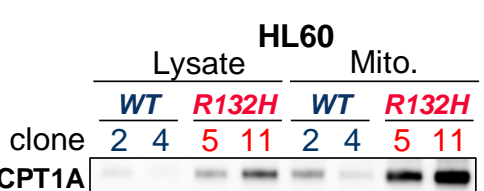
MOLM14
Lysate Mito. WT MUT WT MUT CPT1 dox TFAM $------\sigma$ $-\ldots-28 \mathrm{kDa}$ PCNA

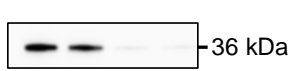

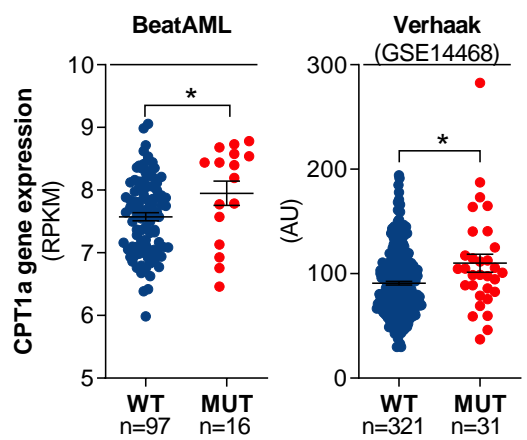

cytosol

Specifically inactive in $\mathrm{R} 132 \mathrm{H}$ cells Specifically active in $\mathrm{R} 132 \mathrm{H}$ cells g

h

\section{BeatAML Verhaak} (GSE14468)

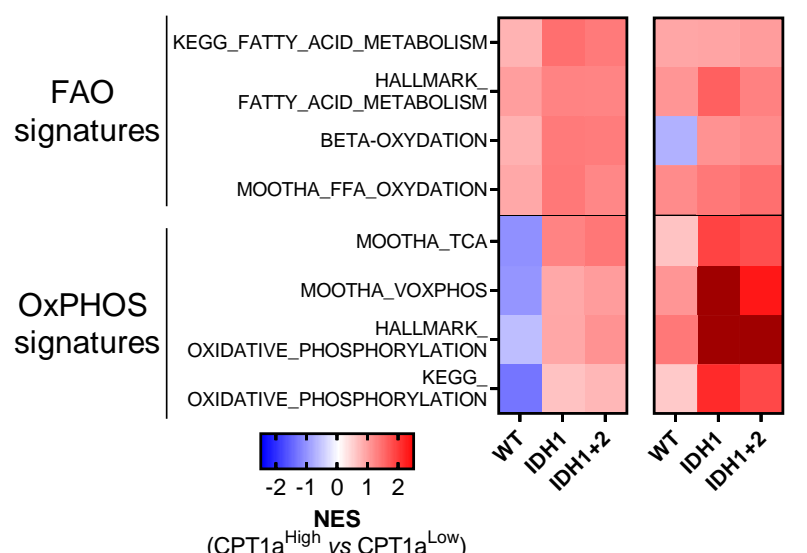

WT R132 WT R132 WT R132

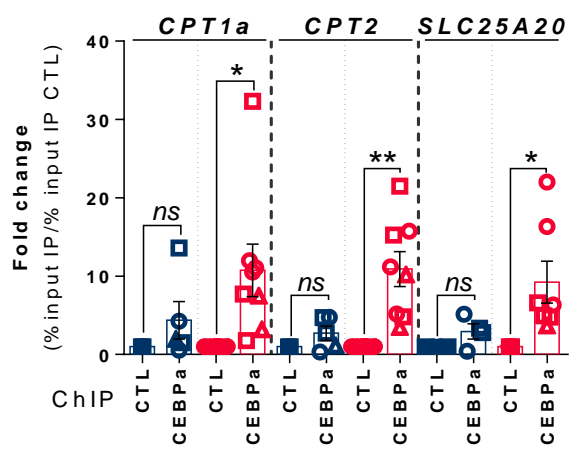




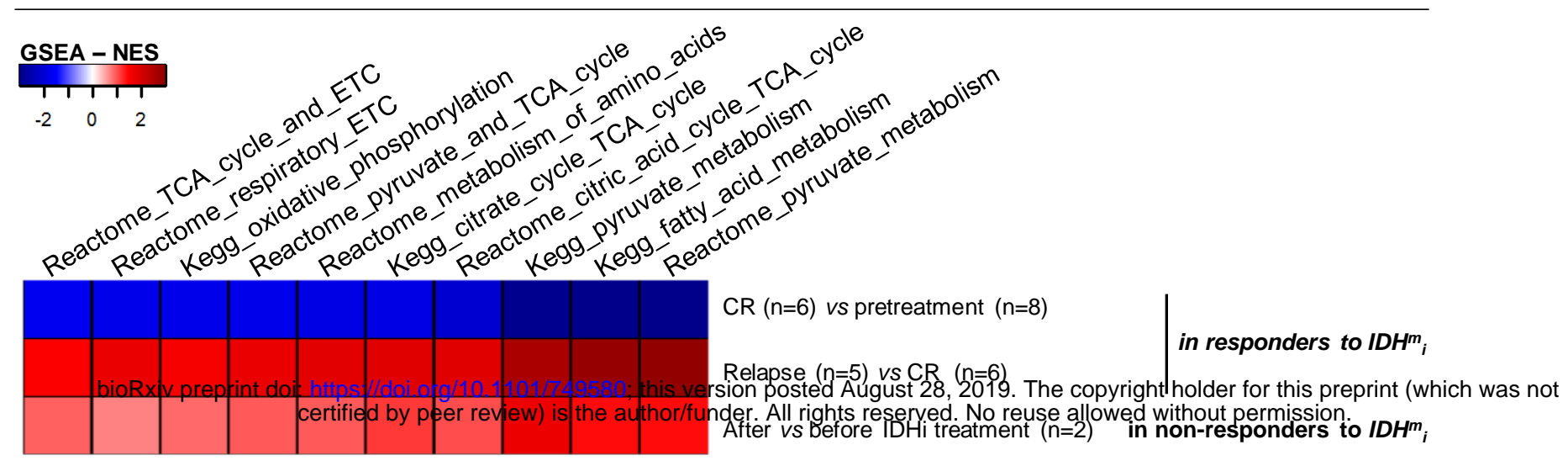

b
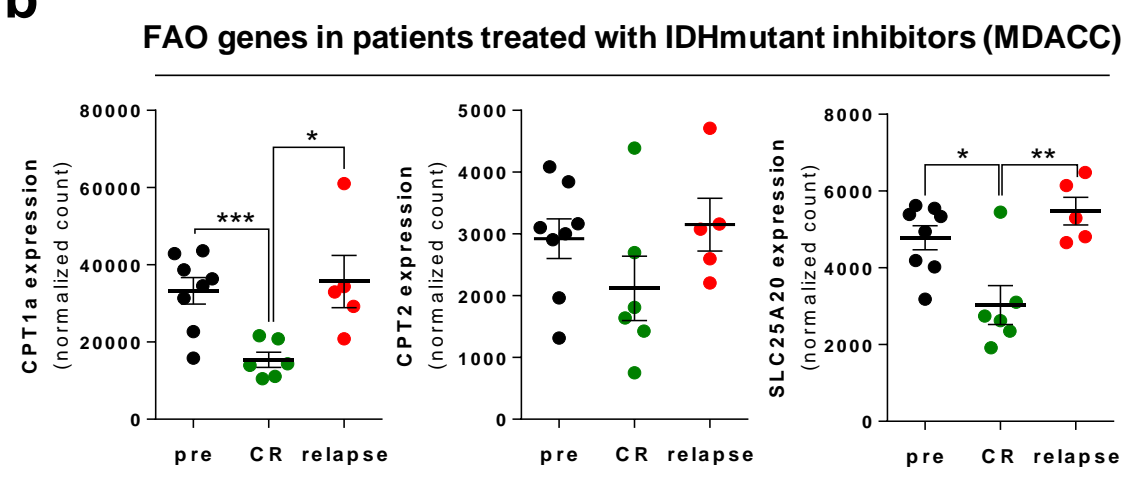

e
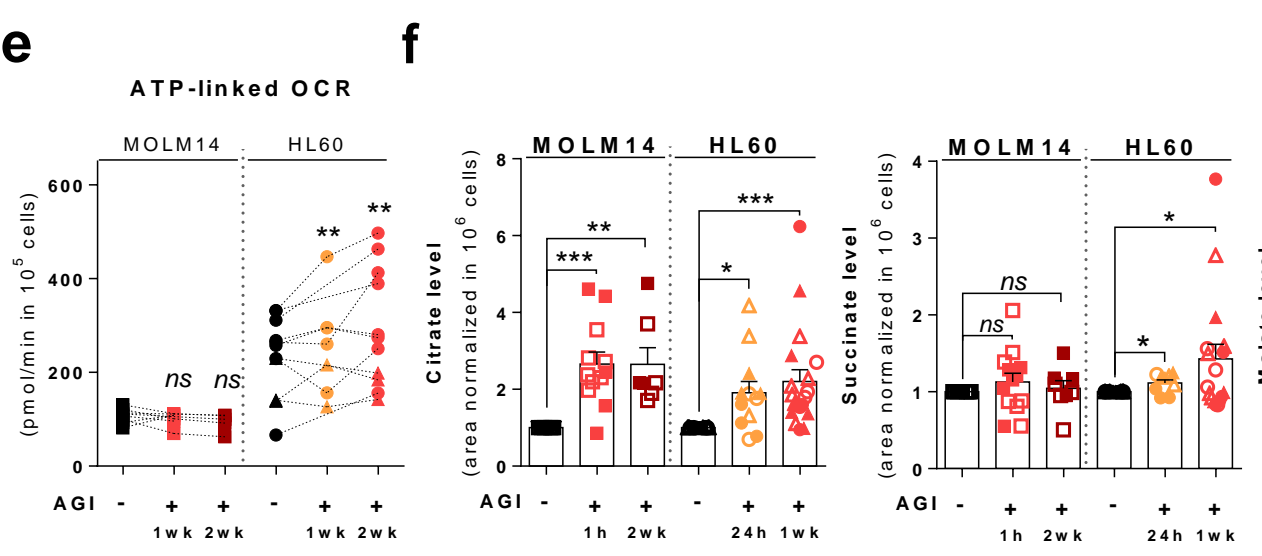

C

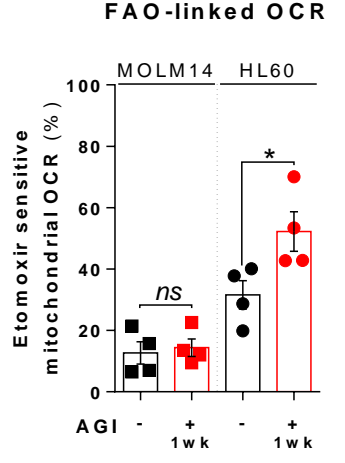

d

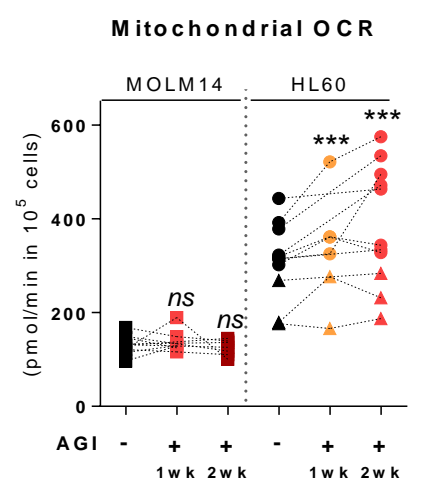




\section{$\mathbf{a}$}

C

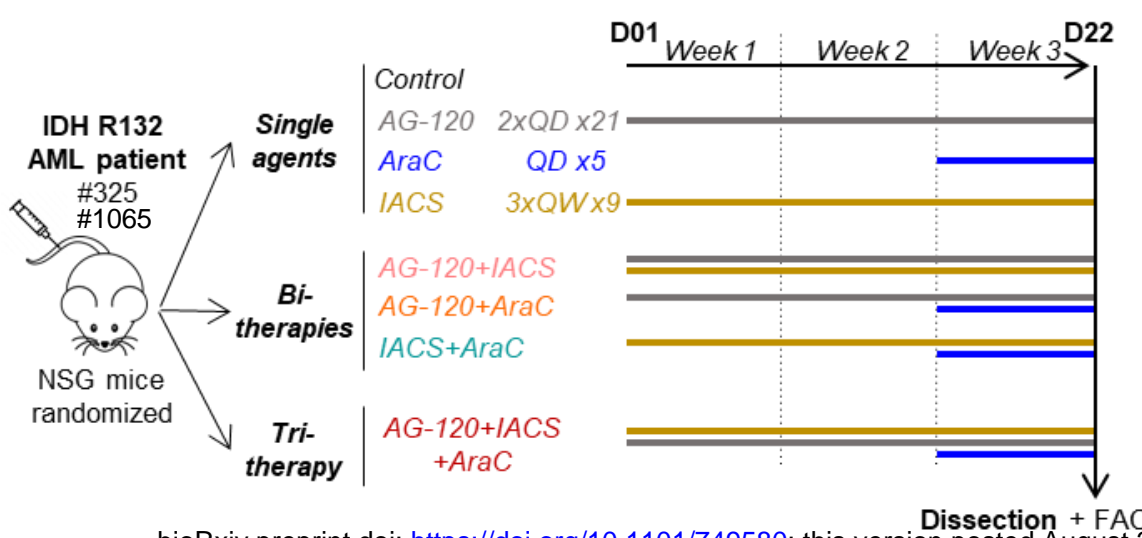

bioRxiv preprint doi: https://doi.org/10.1101/749580; this ver
certified by peer review) is the author/fun

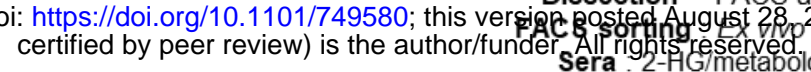

Blood : Counts

$A G-120$ concentration in serum

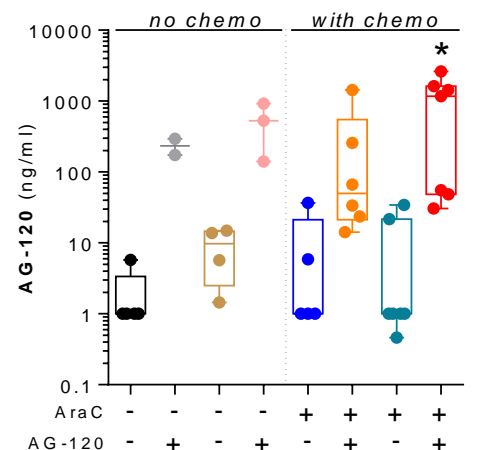
$2 H G$ level in serum

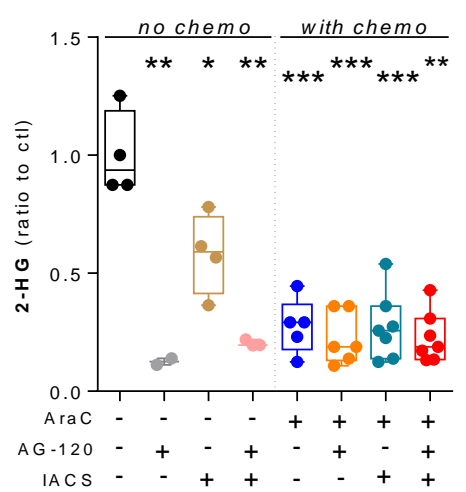

d

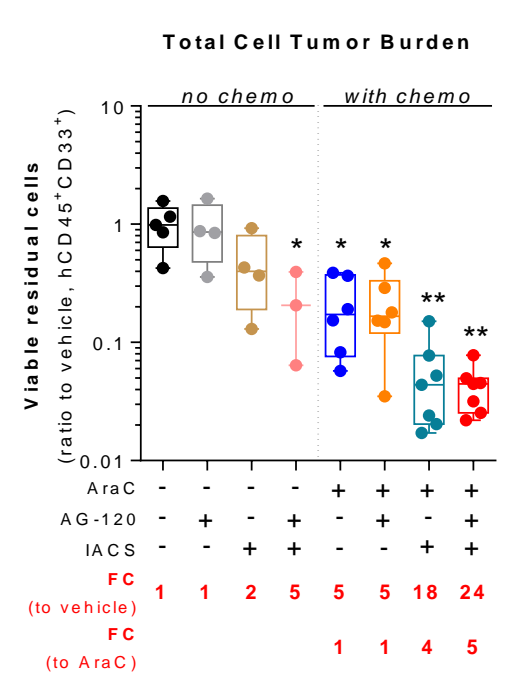

\section{e}

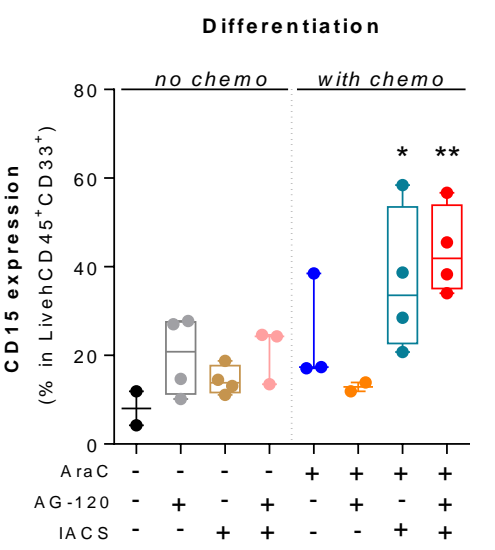

f

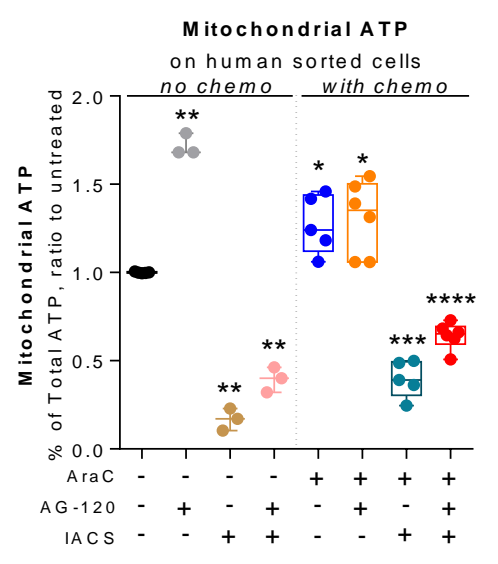

g

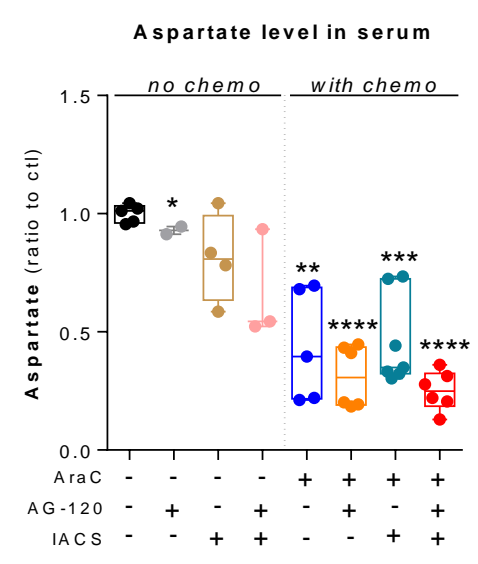

Lactate level in serum

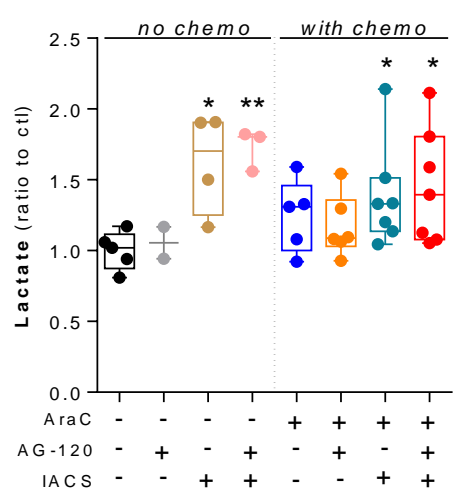

h

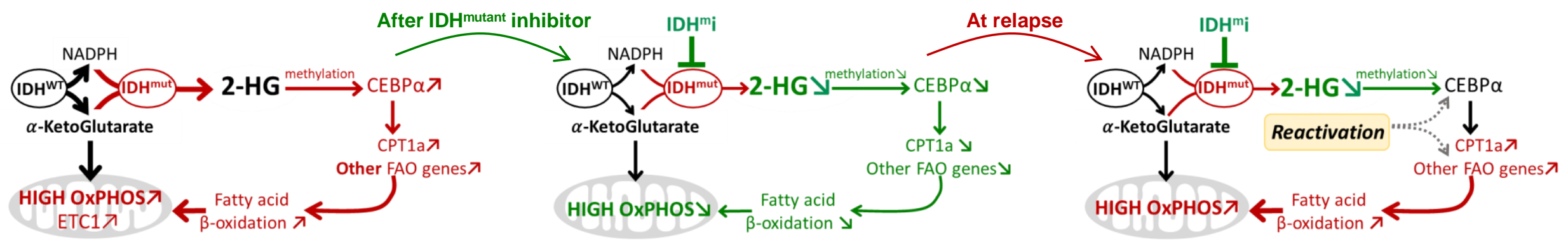

Alternatjue fueling 